\title{
Design and beam dynamic studies of a 30 -MW superconducting linac for an accelerator-driven subcritical system
}

\author{
Bruce Yee-Rendon $\oplus^{*}$ Y Yasuhiro Kondo, Fujio Maekawa, Shin-ichiro Meigo®, and Jun Tamura \\ J-PARC Center, Japan Atomic Energy Agency, Tokai, Ibaraki 319-1195, Japan
}

(Received 12 April 2021; accepted 12 November 2021; published 10 December 2021)

\begin{abstract}
The Japan Atomic Energy Agency (JAEA) is working on the research and development of a 30-MW continuous wave (CW) proton linear accelerator (linac) for the JAEA accelerator-driven subcritical system (ADS) proposal. The linac will accelerate a $20 \mathrm{~mA}$ proton beam to $1.5 \mathrm{GeV}$, using mainly superconducting cavities. The main challenge for an ADS accelerator is the high reliability required to prevent thermal stress in the subcritical reactor; thus, we pursue a robust lattice to achieve stable operation. To this end, the beam optics design reduces the emittance growth and the beam halo through the superconducting part of the linac. First, we simulated an ideal machine without any errors to establish the operation conditions of the beam. Second, we applied element errors and input beam errors to estimate the tolerance of the linac design. Finally, we implemented a correction scheme to increase the lattice tolerance by reducing the beam centroid offset on the transverse plane. Massive multiparticle simulations and a cumulative statistic of $1 \times 10^{8}$ macroparticles have shown that the JAEA-ADS linac can operate with less than $1 \mathrm{~W} / \mathrm{m}$ beam losses in error scenarios.
\end{abstract}

DOI: 10.1103/PhysRevAccelBeams.24.120101

\section{INTRODUCTION}

The long-lifetime and high radiotoxicity of nuclear waste are critical problems that nuclear organizations and countries have been facing for decades. In 1988, the Japan Atomic Energy Research Institute (JAERI), now the Japan Atomic Energy Agency (JAEA), launched the OMEGA project to research and develop an acceleratordriven subcritical system (ADS) [1]. The purpose of the OMEGA program is the partitioning and transmutation of minor actinides. This option leads to a significant reduction of the lifetime and the radioactivity level of nuclear residues; thus, the nuclear waste storage becomes more manageable. Figure 1 shows an overview of the ADS. It comprises a high-power accelerator, a spallation target, and a subcritical nuclear reactor core.

The ADS demands particular requirements for the accelerator operation, such as tens of megawatts of beam power and continuous wave $(\mathrm{CW})$ operation. The latter criteria must be compatible with the reactor operation; as a result, a CW proton superconducting (SC) linear accelerator (linac) is the optimal candidate for this job. Furthermore, as with any high-power machine, hands-on

\footnotetext{
"byee@post.j-parc.jp

Published by the American Physical Society under the terms of the Creative Commons Attribution 4.0 International license. Further distribution of this work must maintain attribution to the author(s) and the published article's title, journal citation, and DOI.
}

maintenance requires strict control of the beam loss below $1 \mathrm{~W} / \mathrm{m}$. The most difficult condition, however, is the high reliability required by the accelerator to avoid thermal stress in the subcritical reactor, which is beyond the current accelerator performance [2-5]. As a first step toward becoming a reliability-oriented accelerator, the Japan Atomic Energy Agency-accelerator-driven subcritical system (JAEA-ADS) linac pursues a robust beam optics design [6].

During the 1990s, the Neutron Science Research Program (NSRP) created the first JAEA-ADS linac design [7-9]. The NSRP was designed to be a multipurpose neutron source that could also be used for ADS research and development. The accelerator had a beam current of $10 \mathrm{~mA}$, a final energy of $1.5 \mathrm{GeV}$, and used normal conducting (NC) cavities in the low-medium relativistic beta $\beta$ range of $0.06(2 \mathrm{MeV})$ to $0.42(100 \mathrm{MeV})$, as well as

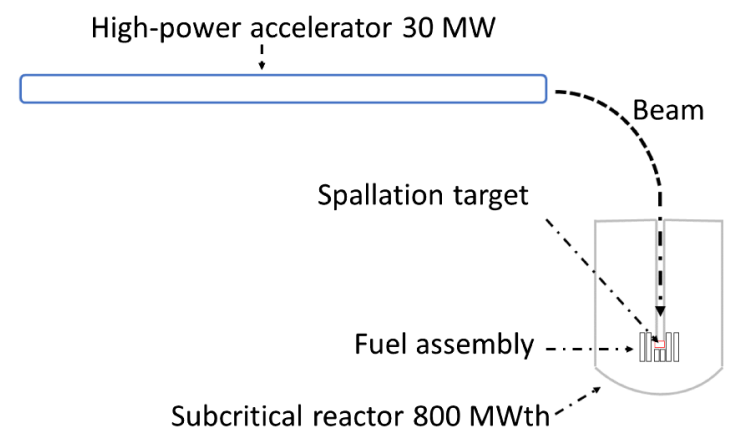

FIG. 1. General scheme for the ADS. 
TABLE I. Main characteristics of the JAEA-ADS linac.

\begin{tabular}{lcc}
\hline \hline Parameter & & Beam trip duration \\
\hline Beam current $(\mathrm{mA})$ & 20 & \\
Proton beam energy $(\mathrm{GeV})$ & 1.5 & \\
Duty factor $(\%)$ & $100(\mathrm{CW})$ & \\
RF frequency $(\mathrm{MHz})$ & $162 / 324 / 648$ & \\
Beam loss $(\mathrm{W} / \mathrm{m})$ & $<1$ & \\
Beam trips per year [3] & $2 \times 10^{4}$ & $\leq 10 \mathrm{~s}$ \\
& $2 \times 10^{3}$ & from $10 \mathrm{~s}$ to 5 min \\
& 42 & $>5 \mathrm{~min}$ \\
\hline \hline
\end{tabular}

SC cavities in the high- $\beta$ section. Later, the NSRP was combined with the KEK Japan Hadron Project (JHP) [10] to become the J-PARC project [11] although, the J-PARC linac achieves the final energy using $\mathrm{NC}$ cavities rather than SC cavities. The development of SC cavities for the medium energy range has contributed to increasing accelerating efficiency and beam power of ion and proton linacs over the last two decades [12,13]. As a result, JAEA relaunched a campaign for the ADS project to construct an SC proton linac [14-17]. In comparison to the NSRP linac, the present linac has double the beam current. Consequently, the main linac section must be completely redesigned from $2.5 \mathrm{MeV}$ to $1.5 \mathrm{GeV}$. The main features of the JAEA-ADS linac are listed in Table I, which are based on the specifications of an 800-MW thermal power ADS [18].

The SC cavity can have large apertures, which reduce the probability of beam loss, in addition to its capacity to $\mathrm{CW}$ operation. Due to our experience in the design and operation of NC RFQ at JAEA, we decided to use NC cavities in the injector section for the rf quadrupole (RFQ) and medium-energy beam transport (MEBT).

The JAEA-ADS linac is shown in Fig. 2. It is composed of an NC section and SC one, the so-called main linac. The $\mathrm{NC}$ section contains an electron cyclotron resonance ion source, a low-energy beam transport, an RFQ, and a MEBT. The main linac consists of five SC cavity sections: one half-wave resonator (HWR), two single-spoke resonators (SSR), and two five-cell elliptical resonators (EllipR).

The present linac design substituted the drift tube linac (DTL) of the NSRP with HWRs and SSRs. The eight different EllipR families were also reduced to two, and the energy transitions between different cavity sections were optimized to reduce the number of cavities. In addition, at NSRP, operation rf frequencies marked as $f_{\mathrm{rf}} \mathrm{s}$ of 200 and $600 \mathrm{MHz}$ were changed to 162,324 , and $648 \mathrm{MHz}$, respectively. In the medium-high $\beta$ sections, the frequency jumps provide a high accelerating gradient. We selected the start $f_{\text {rf }}$ of $162 \mathrm{MHz}$ to reduce transverse deflecting rf kick at the low energy. Because of our experience in the J-PARC linac, we chose $f_{\mathrm{rf}} \mathrm{s}$ of 324 and $648 \mathrm{MHz}$.

The beam dynamics design and particle simulations, as well as error studies, are reported in this work. The details of the linac design are discussed in Sec. II. Section III presents the result of beam simulations for the ideal machine and error cases. Section IV contains the conclusions.

\section{LINAC DESIGN}

\section{A. Beam operation conditions}

Because the JAEA-ADS linac runs at a higher intensity than other ADS projects $[19,20]$, it is subjected to strong space-charge effects and strict beam loss rate limitations. The space-charge effects in the lattice were analyzed by the tune depression $\sigma / \sigma_{0}$, where $\sigma$ is the phase advance with space-charge and $\sigma_{0}$, with zero current. Moreover, the $\sigma_{0} \mathrm{~s}$ must be lower than 90 deg in all axes to avoid parametric resonances [21].

The linac design's tune depressions are plotted in Fig. 3. The average was 0.59 on the transverse direction and 0.54 on the longitudinal. This shows that despite the medium beam current of $20 \mathrm{~mA}$, the dynamic of our linac is spacecharge dominated. The reason for this is the lower focusing force of the SC linac, especially in the low- $\beta$ region, as compared to a NC linac such as DTL.

Figure 4 is the Hofmann chart [22] for the main linac. In general, there are two possibilities for the operation point:

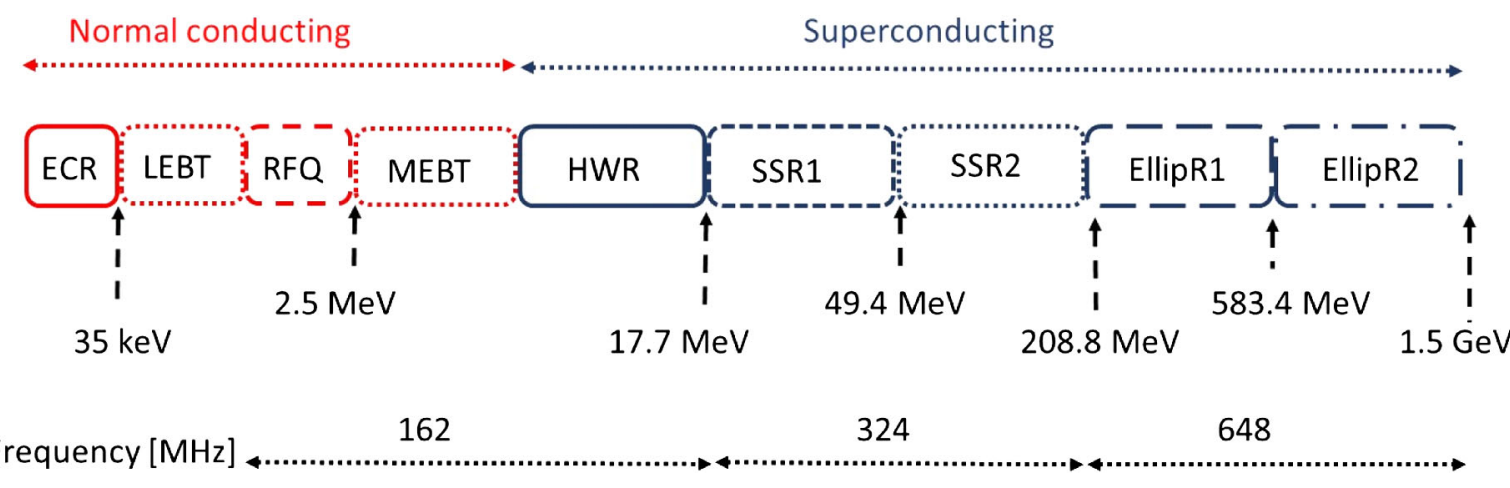

FIG. 2. Layout of the JAEA-ADS linac. 


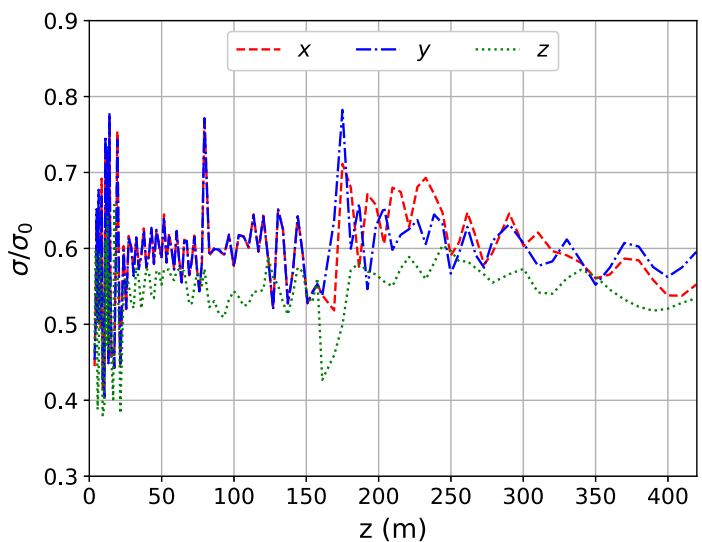

FIG. 3. Tune depressions along the main linac.

$\sigma_{z} / \sigma_{x y}<1$ or $\sigma_{z} / \sigma_{x y}>1$, where $\sigma_{x y}$ is the average phase advance between the $x$ axis and $y$ axis. We choose to operate in the $\sigma_{z} / \sigma_{x y}<1$ region because we accessed stronger transverse focusing than the other option, $\sigma_{z} / \sigma_{x y}>1$. As a result, we achieve better control of the space-charge dominant beam [14]. The color points represent the different superconducting (SC) cavity periods; periods that belong to the same section have the same color.

Furthermore, to avoid emittance transmission between axes, we followed equipartitioning beam [23],

$$
\frac{\sigma_{x y}}{\sigma_{z}}=\frac{\varepsilon_{\text {norm,rms }, \mathrm{z}}}{\varepsilon_{\text {norm,rms }, \mathrm{xy}}},
$$

where $\varepsilon_{\text {norm,rms }}$ is the normalized root-mean-square emittance. The $\sigma_{0 x y}$ values are adjusted by magnets; the $\sigma_{0 z}$ by

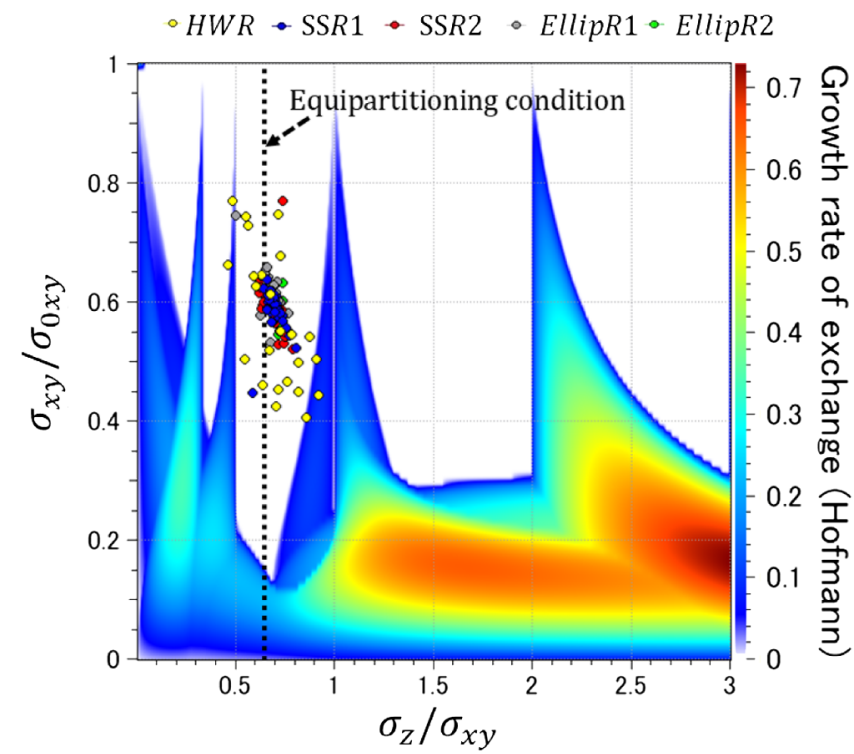

FIG. 4. Hofmann chart shows the work points for each of the different SC periods of the main linac, the equipartitioning condition (black dotted line) and the instabilities areas of the second-, third-, and fourth-order modes. the rf cavities. In our case, the $\varepsilon_{\text {norm,rms }} \mathrm{s}$ at the end of the MEBT were 0.23 and $0.38 \pi \mathrm{mm}$ mrad for the transverse and longitudinal directions, respectively. However, as a first step, we used $\sigma_{0 x y} / \sigma_{0 z}$ equal to 1.4. Beam simulations indicated that 1.4 at the HWR section, 1.36 at SSRs, and 1.36 at ElllipRs provided better control of the emittance growth; therefore, we used those values for each of the sections. It is worth noting that the Hofmann chart predicted zero emittance exchange at working points other than those provided by equipartitioning. The equipartitioning condition, on the other hand, nevertheless produced the best linac performance.

\section{B. Beam optics}

\section{MEBT}

The design of the superconducting linac optics starts at the MEBT. This section matches the beam from the end of the RFQ to the first SC section controlling the emittance growth. The space-charge is the main source of emittance growth and beam halo at beam energy of $2.5 \mathrm{MeV}$. Thus, a regular compact optic design is sought, which enables uniform focusing in all axes; however, the MEBT must also contain sufficient diagnostic elements for beam tuning. Using four quadrupoles and two bunchers, an early MEBT design [24] kept the emittance growth below $20 \%$.

We redesigned the MEBT by adding two more quadrupoles, four scrappers, and beam diagnostics to improve the beam quality in the downstream sections after updating the HWR section in the main linac. Figure 5 presents the

(a) Old model

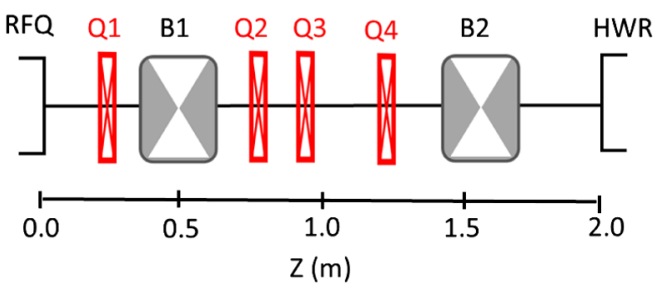

(b) Present model

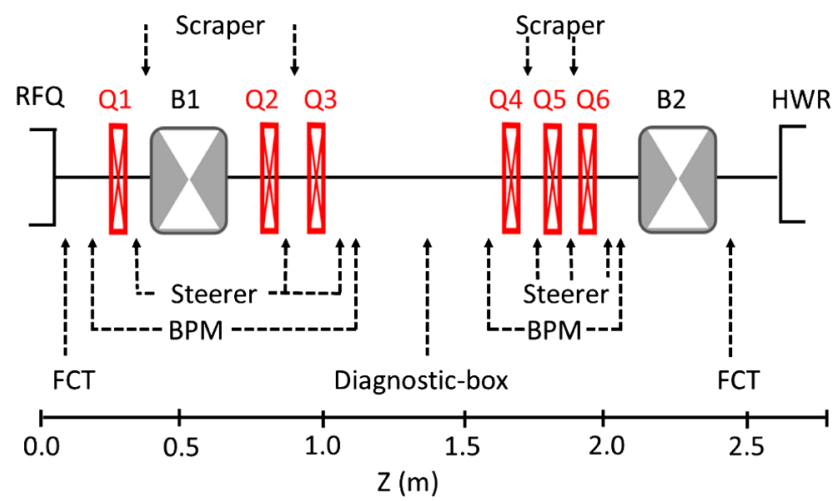

FIG. 5. MEBT layouts for the JAEA-ADS. The upper section depicts the previous design [24], while the lower section depicts the current configuration. 
TABLE II. Parameters of the MEBT elements. Q gradients with a positive sign indicate that the beam is focused on the $x$ direction.

\begin{tabular}{lcc}
\hline \hline Element $^{\mathrm{a}}$ & Length $(\mathrm{mm})$ & Magnet gradient $(\mathrm{T} / \mathrm{m}) /$ voltage $(\mathrm{kV})$ \\
\hline Q1 & 60 & 11.19 \\
$\mathrm{~B} 1$ & 300 & 80.5 \\
Q2 & 60 & -15.5 \\
Q3 & 60 & 13.1 \\
Q4 & 60 & -10.7 \\
Q5 & 60 & 17.4 \\
Q6 & 60 & -10.5 \\
B2 & 300 & 125.2 \\
\hline \hline
\end{tabular}

${ }^{\mathrm{a}} \mathrm{Q}$ stands for quadrupole and B for buncher cavity.

previous version on the top and the present version on the bottom. The MEBT length grew $0.5 \mathrm{~m}$ compared to the previous, about $25 \%$; however, the emittance growth is less than 5\%. Table II summarizes the MEBT's matching elements.

A notable implementation was the use of a scraping system to reduce the beam halo. The MEBT has four scrapers assemblies: two horizontal and two vertical. After the first and fifth quadrupoles, the horizontal ones are found. The vertical scrappers are after the second and fourth quadrupoles in the same way. To avoid a large reoptimization of the downstream of the lattice, the amount of collimated beam was chosen to minimize the maximum transverse beam size without compromising the beam core. The jaws' positions and apertures were selected using TraceWin [25] beam tracking simulations. The scraper jaws were optimized to accept up to $100 \mathrm{~W}$ per assembly and the entire setup removed less than $1 \%$ of the beam. Figure 6 shows the results of beam simulations using the scrapers for different beam envelope fractions. Furthermore, Fig. 7 presents the transverse profile at the end of the MEBT with and without beam scraping
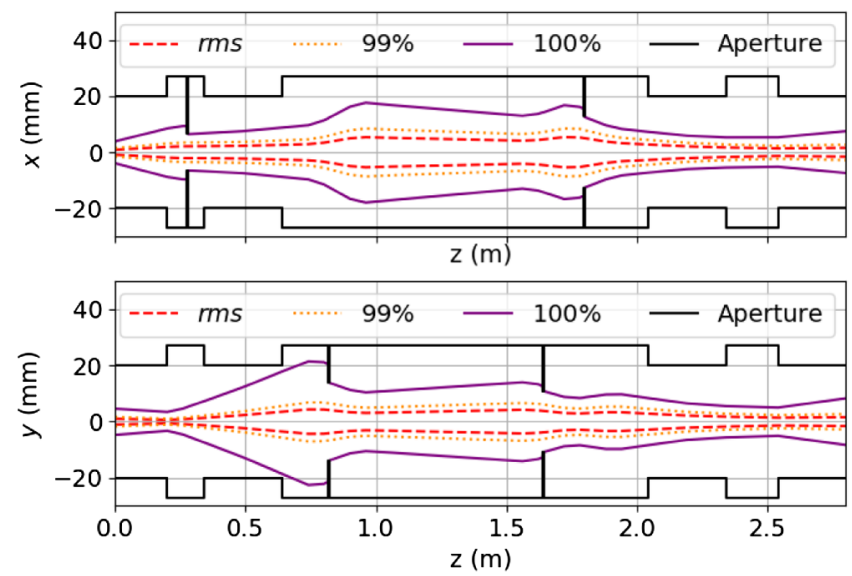

FIG. 6. Transverse beam envelopes for different beam fractions along the MEBT.
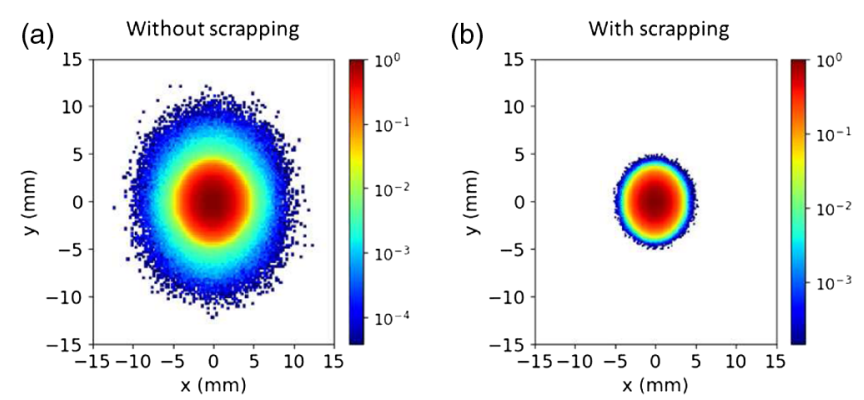

FIG. 7. Transverse beam distribution with and without scraping at the end of the MEBT.

(right and left). The maximum transverse beam radius was lowered from 12 to $5 \mathrm{~mm}$ without affecting the beam core.

A correction scheme based on the SARAF MEBT [26] was also implemented. The first to third quadrupoles were surrounded by a pair of beam position monitors (BPM), while the last three quadrupoles were flanked by a second pair of BPM. A steering magnet is also attached to each quadrupole to restore the beam centroid from lattice errors.

\section{Main linac}

To achieve a robust beam optic design, the lattice must have strict beam loss control, a simple configuration, and operate with derated SC cavities to reduce the failure risks. In addition, we want to reduce the number of SC cavities and the linac length to minimize the operational cost.

In the main linac, the proton beam $\beta$ changed from 0.07 to 0.923 . The types of SC cavities were selected based on the energy region in which they present the best performance. EllipRs, for example, are the common choice for $\beta>0.5$. HWR and SSR were chosen for their $f_{\text {rf }}$ range and high-performance for a $\beta$ value between 0.07 to 0.5 . Then, we optimized the number of different SC cavities, $N_{\text {cell }}$, and geometrical beta $\beta_{g}$ to achieve the greatest transit time factor $T$ on the linac's energy range. The $T$ described in Refs. [27,28], which assumes a sinusoidal electric field on the cavity axis, is used in this case:

$$
\begin{aligned}
T= & \frac{\beta}{2 N_{\text {cell }}}\left\{\frac{\sin \left[\pi N_{\text {cell }}\left(\beta-\beta_{g}\right) /(2 \beta)\right]}{\beta-\beta_{g}}\right. \\
& \left.-\frac{(-1)^{N_{\text {cell }}} \sin \left[\pi N_{\text {cell }}\left(\beta+\beta_{g}\right) /(2 \beta)\right]}{\beta+\beta_{g}}\right\} .
\end{aligned}
$$

Figure 8 displays the $T$ normalized to its maximum value, with the transition energies shown by black dotted lines. Two types of SSR and EllipR were chosen to achieve higher values of $T$ in the energy operation range. The final transition energies were readjusted to achieve stability conditions and reduce the linac's length.

CST MICROWAVE STUDIO [29] and SUPERFISH [30] were used to create electromagnetic models of the SC cavities. Only the latter was utilized for the EllipR cavities. The SC 


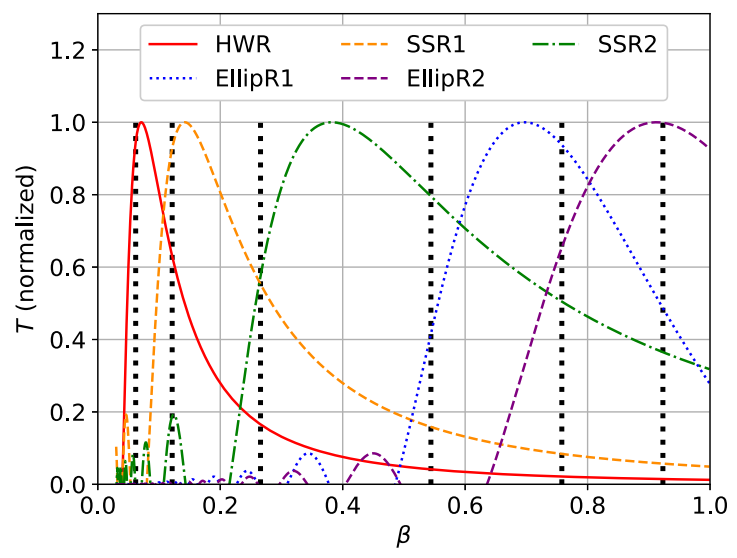

FIG. 8. Transient time factor $T$ for each SC cavity as a function of relativistic $\beta$. The black dotted lines indicate the energy transition between the types of cavities.

models were adjusted by minimizing the ratios of the peak surface electromagnetic fields, $E_{p k}$ and $B_{p k}$, to the accelerating gradient $E_{\text {acc. }}$. To reduce the likelihood of field emission or quench, the $E_{p k}$ on the cavity's surface was limited to $30 \mathrm{MV} / \mathrm{m}$; as a result, the $B_{p k}$ for all the cavities was less than $60 \mathrm{mT}$. Additionally, by maximizing the values of the geometric shunt impedance $R / Q$ and the geometric factor $G$ [14], the power dissipation in the cavity's walls was reduced. Table III presents a summary of the SC cavities. The aperture of the cavity was chosen to meet the requirements of the beam optics.

The optics design of the main linac was optimized to operate with beam loss of less than $1 \mathrm{~W} / \mathrm{m}$. To this end, the JAEA-ADS lattice was oriented to minimize emittance growth due to space-charge to reduce particle loss and beam halo. The design was carried out with the programs GenLinWin [31] and TraceWin. First, GenLinWin creates the lattice for each SC period, tunes the cavity setting, and generates the TraceWin input. Then, TraceWin set up the transverse parameters, made the matching, produced envelope and multiparticle simulations, simulated error scenarios, and applied compensation schemes. The beam optics began with the lattice configuration of the different SC sections.

TABLE III. Summary of the JAEA-ADS SC cavities.

\begin{tabular}{lccccc}
\hline \hline Parameters & HWR & SSR1 & SSR2 & EllipR1 & EllipR2 \\
\hline$f_{\text {rf }}(\mathrm{MHz})$ & 162 & 324 & 324 & 648 & 648 \\
$\beta_{g}$ & 0.08 & 0.16 & 0.43 & 0.68 & 0.89 \\
$N_{\text {cell }}$ & 2 & 2 & 2 & 5 & 5 \\
Aperture $(\mathrm{mm})$ & 40 & 40 & 40 & 80 & 94 \\
Maximum $E_{\text {acc }}(\mathrm{MV} / \mathrm{m})$ & 7.12 & 6.60 & 8.45 & 13.82 & 14.21 \\
$E_{p k} / E_{\text {acc }}$ & 4.21 & 4.50 & 3.55 & 2.17 & 2.11 \\
$B_{p k} / E_{\text {acc }}[\mathrm{mT} /(\mathrm{MV} / \mathrm{m})]$ & 3.41 & 6.68 & 5.13 & 4.22 & 4.07 \\
$R / Q(\Omega)$ & 285 & 212 & 285 & 443 & 619 \\
$G(\Omega)$ & 59 & 64 & 129 & 208 & 256 \\
\hline \hline
\end{tabular}

The space-charge effects are larger in the low- $\beta$, such as HWR and SSR sections, than in the high- $\beta$ EllipR regions. A short focusing period is desired to regulate the emittance growth. Instead of NC quadrupoles, an SC solenoid-cavity per period configuration was adopted to produce a compact lattice period. The HWR cavities were previously housed in a single cryomodule to maintain lattice periodicity [14-17]. As a result, the design was able to attain a good beam performance. However, we abandoned that plan since it would result in an $18 \mathrm{~m}$ long cryomodule that would be difficult to construct, operate, and maintain. Thus, we split into three cryomodules: one with nine periods and two with eight. The nine cavities and eight cavities cryomodules have lengths of 6.6 and 6 meters, respectively. On the contrary, the SSR sections contain more than one cavity per focusing period and one cryomodule per period.

Because of their focusing strength, $\mathrm{NC}$ quadrupoles were chosen for the focusing-defocusing-cavity configuration in the high- $\beta$ region. Furthermore, NC quadrupoles align more precisely than SC quadrupoles.

Mechanical restrictions controlled the drift spaces between elements. We include gaps of $400 \mathrm{~mm}$ for cryomodule interconnections on both sides of the lattice period for this purpose. The lattice configuration for the different SC sections is shown in Fig. 9.

The cavity number per period was chosen by comparing the beam simulation results with different configurations. The optimization goal was to reduce the total amount of cavities in the main linac without decreasing the optic's performance. For instance, the cavity number per period at the SSR2 was varied from two to four. As a result, three cavities per period reduced the total number of cavities without increasing the beam loss and the emittance growth. We applied similar studies for SSR1 and EllipRs sections.

Figure 10 presents the synchronous phase $\phi_{s}$ (top) and the corresponding effective accelerating gradient $E_{0} T$ of the cavities (bottom) of the final linac design. The $z$ position along the linac is represented by the horizontal axis. The MEBT region is not included in this section. The blue and red vertical lines highlight the transition between different SC parts. The red lines indicate the location of the frequency jump. The initial $\phi_{s}$ and $\sigma_{0 z}$ at the beginning of the main linac are input values for GenLinWin.

At the HWR entrance, $\phi_{s}$ was set to -60 deg to provide adequate phase acceptance. On the other hand, $\sigma_{0 z}$ is constrained by $\sigma_{0 x y}$ according to the equipartitioning condition. We chose the maximum $\sigma_{0 z}$ equals $57 \mathrm{deg}$ through the whole linac; subsequently, the maximum from $\sigma_{0 x y}$ is $80 \mathrm{deg}$, as shown in Fig. 11 (top). The phase advances obtained after applying beam matching are shown in Fig. 11; as a result, some periods have $\sigma_{0 x y}$ more than $80 \mathrm{deg}$, but less than $90 \mathrm{deg}$. GenLinWin calculates the corresponding phase advance per unit length $\sigma_{0 z} / L_{\text {period }}$ from the initial $\sigma_{0 z}=57 \mathrm{deg}$, and $E_{0} T$ was adjusted using the following formula: 
(a)

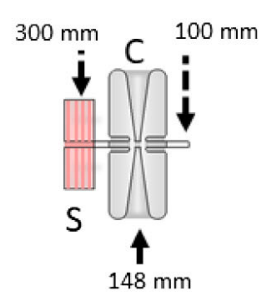

(b)

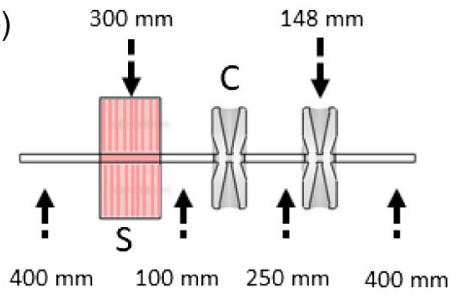

(c)

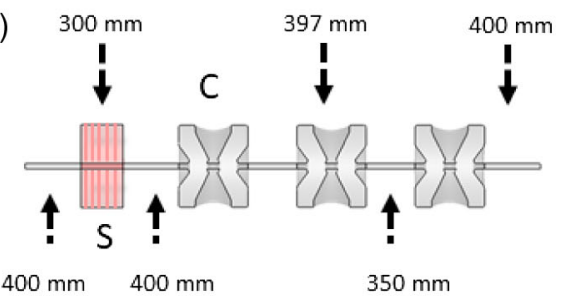

(d)

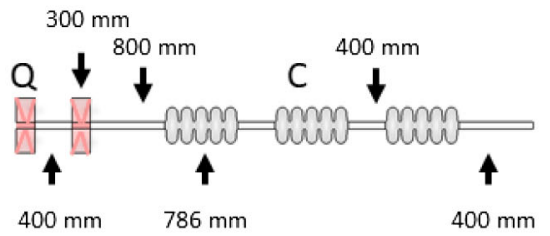

(e)

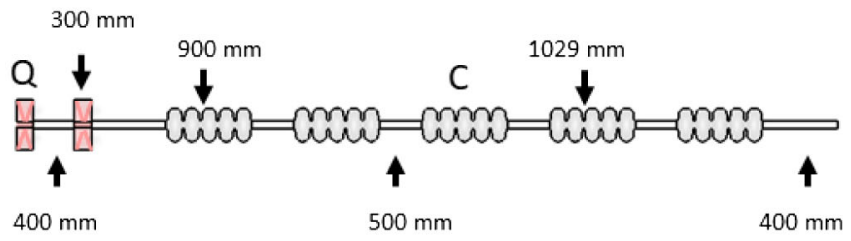

$\mathrm{S}=$ Solenoid $\mathrm{Q}=$ Quadrupole, and $\mathrm{C}=$ Cavity

FIG. 9. The different SC cavity period lattices for the JAEA-ADS: (a) for the HWR section, (b) and (c) correspond to the spokes sections SRR1 and SSR2, respectively, and (d) is for EllipR1 and (e) for EllipR2.

$$
\left(\sigma_{0 z} / L_{\text {period }}\right)^{2}=\frac{2 \pi q E_{0} T \sin \left(-\phi_{s}\right)}{m_{0} c^{2} \beta_{s}^{3} \gamma_{s}^{3} \lambda}
$$

where $L_{\text {period }}$ is the period length, $m_{0} c^{2}$ is the rest mass, $\beta$ and $\gamma$ are the relativistic parameters, and $\lambda=c / f_{\mathrm{rf}}$.

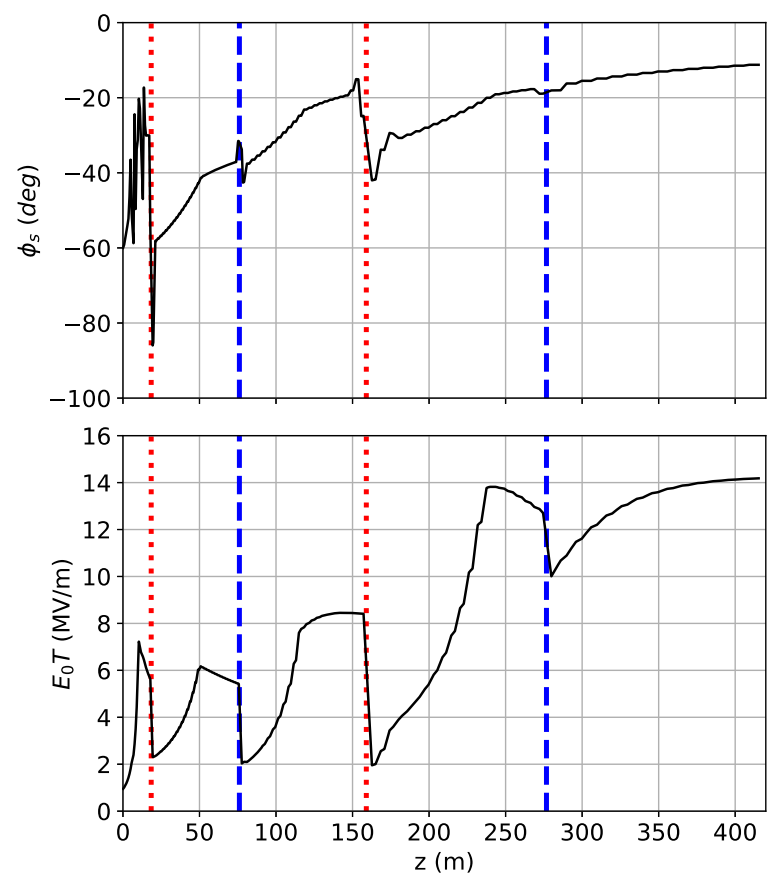

FIG. 10. Synchronous phase (top) and the accelerating gradient (bottom) of the SC cavities. The vertical lines show the transition between different SC sections. The red dotted lines indicate the region of frequency jumps and the change of SC regions.
To achieve a bigger energy gain, GenLinWin increased $\phi_{s}$ up to the limit of each section while maintaining phase acceptance by following the phase damping law:

$$
\phi_{s, b}=\phi_{s, a} \times\left(\frac{\left(\beta_{s} \gamma_{s}\right)_{a}}{\left(\beta_{s} \gamma_{s}\right)_{b}}\right)^{3 / 4}
$$
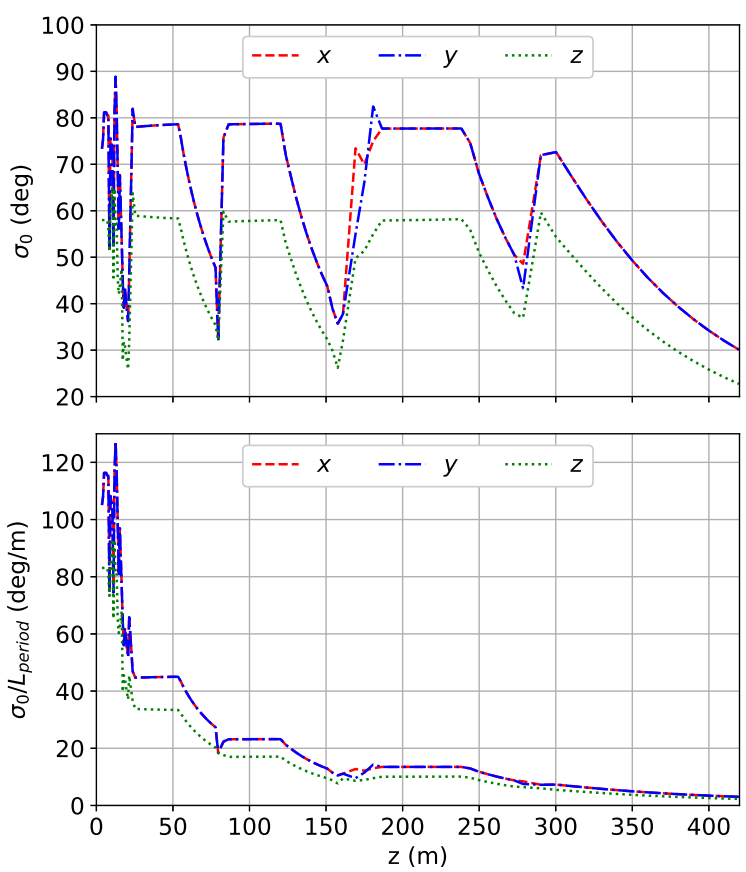

FIG. 11. Phase advance per period (top) and per unit length (bottom). The $x$ axis is represented by the red solid line, the $y$ axis by the blue dashed line, and the $z$ axis by the green dotted line. 
where $\beta_{s}$ and $\gamma_{s}$ are the relativistic parameters of the synchronous particle at the start point $a$ and some later point $b$.

$\phi_{s}$ was limited to a maximum value of $-30 \mathrm{deg}$ in the prior design's HWR section to avoid a bottleneck in the phase acceptance. Due to the breaking of the periodicity as a result of separating the HWR cryomodule into three, the $\phi_{s}$ was modified to manage the beam performance. As illustrated in Fig. 10 (top), this effect resulted in a nonsmooth evolution of $\phi_{s}$ at HWR. GenLinWin increases the $\phi_{s}$ and $E_{0} T$ without exceeding the $\sigma_{0 z}$ limit until this value is reached. $\phi_{s}$ reaches its maximum at some point. The transition energy from the current segment to the next was then optimized by the algorithm. The optimization goal was to decrease the overall number of cavities in the linac. $E_{0} T$ is adjusted in the final parts before the transition to ensure that the $\sigma_{0 z} / L_{\text {period }}$ goes smoothly into the next segment. We want $\sigma_{0} / L_{\text {period }}$ to have an adiabatic evolution through the linac in all three directions to limit emittance growth [32], and we also want to use an equipartitioning beam. Thus, $\sigma_{0 x y}$ is calculated using the $\sigma_{0 x y} / \sigma_{0 z}=1.4$.

The phase acceptance is maintained at the frequency jump by adjusting $\phi_{s}$ in proportion to the frequency jump, as illustrated by the red dotted lines in Fig. 10, where the absolute value of $\phi_{s}$ increased twice as much as in the preceding section. This condition retains longitudinal acceptance, hence lowering emittance growth and increasing beam transmission [33].

Figure 12 shows the longitudinal acceptance at the entrance of the main linac, white area, obtained by beam tracking simulations. The gray area indicates the initial location of the particle that becomes lost during beam transportation. The solid red ellipse at the main linac's entry represents one $\varepsilon_{\text {norm,rms,z }}(0.07 \pi \mathrm{MeV} \mathrm{deg})$ longitudinal phase-space area of the distribution considered to be the input beam specified in Sec. III. The empty red ellipse is the

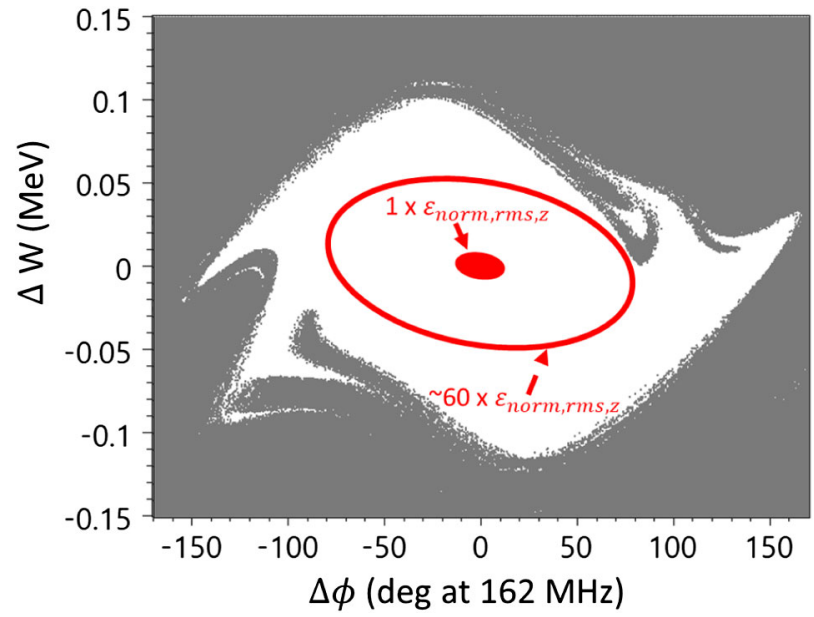

FIG. 12. Longitudinal acceptance at the entrance of the main linac. maximum area of the same ellipse parameters transported through the linac without beam losses. The longitudinal acceptance was guaranteed by applying the continuous phase acceptance condition, resulting in an equivalent area of 60 times the input $\varepsilon_{\text {norm,rms,z }}$.

At the beginning of the SSR1 section, $E_{0} T$ was reduced to prevent $\sigma_{0 z}$ exceeding $57 \mathrm{deg}$ despite the decrement of $\phi_{s}$ described above. Then, $\phi_{s}$ evolved according to Eq. (4) and $E_{0} T$ was adjusted from $\sigma_{0 z} / L_{\text {period. }}$. After $E_{0} T$ reached the limit corresponding to $E_{\text {acc }}$ listed in Table III, it was kept from exceeding the limit. The $\phi_{s}$ ramping was done maintaining the separatrix area, and adjustments were made to ensure smooth continuity of the $\sigma_{0 z} / L_{\text {period }}$ with the proceeding section. Finally, with SSR2, the transition energy was chosen to provide a smooth $\sigma_{0 z} / L_{\text {period }}$ between the two sections and reduce the number of cavities.

The same technique was used for the SSR2 to EllipR1 sections as it was for the HWR to SSR1. Finally, the passage from EllipR1 to EllipR2 followed an analogous process as SSR1 to SSR2. Furthermore, in the EllipR2 region, the reduction of $E_{0} T$ was not required at the entrance to fulfill the limit of $\sigma_{0 z}$. The selected numbers of cavities per section in the main linac are listed in Table IV.

Table V summarizes the magnet features obtained for the main linac. Beam optics and engineering factors influenced the magnet parameters. The solenoids, for example, have a $40 \mathrm{~mm}$ aperture, a $300 \mathrm{~mm}$ length, and a maximum magnetic field of up to $4.2 \mathrm{~T}$. These features match those of other high-intensity linacs [34]. The properties of the quadrupoles are likewise compatible with those of earlier efforts [35]. Quadrupole's apertures were chosen to include roughly $15 \mathrm{rms}$ beam size to reduce beam losses, and a maximum gradient of $8.06 \mathrm{~T} / \mathrm{m}$, which is feasible with the JAEA staff's current experience.

Reduced emittance growth also requires beam matching. The smoothness of the phase advance and synchronous phase, on the other hand, is affected. TraceWin was used to change the magnet's gradients and the cavity's phase of the element around the transition region to match the beams. It was particularly difficult to achieve all of the design parameters, such as a smooth envelope, low emittance increase, and $\sigma_{0}<90 \mathrm{deg}$ when beam matching between the HWR cryomodules. Drift spaces were also added to all

TABLE IV. Summary of the number of cavities in the main linac.

\begin{tabular}{lc}
\hline \hline Section & Number of cavities \\
\hline HWR & 25 \\
SSR1 & 66 \\
SSR2 & 72 \\
EllipR1 & 60 \\
EllipR2 & 70 \\
\hline \hline
\end{tabular}


TABLE V. Summary of the magnet features in the main linac.

\begin{tabular}{lcc}
\hline \hline Parameters & Solenoids & Quadrupoles \\
\hline Type & SC & NC \\
Aperture $(\mathrm{mm})$ & 40 & 70 \\
Effective length $(\mathrm{mm})$ & 300 & 300 \\
Magnetic field $(\mathrm{T})$ & 1.5 to 4.2 & \\
Gradient $(\mathrm{T} / \mathrm{m})$ & & 0.9 to 8.6 \\
Total number & 83 & 70 \\
\hline \hline
\end{tabular}

${ }^{\mathrm{a}}$ The additional magnets for the matching are included.

matched sections to achieve smooth envelopes. Furthermore, one extra solenoid was necessary for the HWR and SSR1 sections to match; similarly, one pair of doublet quadrupoles was required for the SSR2 and EllipR1 sections to match. Consequently, the phase advance was distorted in the matching areas. The horizontal $\sigma_{0}$ increased near the entry of EllipR 1 after that, although it remained less than $90 \mathrm{deg}$.

\section{BEAM DYNAMICS STUDIES}

\section{A. Ideal machine}

TraceWin software was used to conduct multiparticle tracking tests from the MEBT to the linac's end. Controlling beam loss below the hands-on maintenance limit of $1 \mathrm{~W} / \mathrm{m}$ is a priority task for the ADS linac to achieve steady operation. Figure 13 shows the beam energy and the corresponding beam loss rate limit along the whole linac.

A beam distribution of $10^{8}$ macroparticles is ideal for making an accurate calculation of the beam losses at the linac's end. However, due to a computational resource constraint, we decided that a beam distribution of $10^{7}$ macroparticles would suffice for a proper beam loss estimation until the end of EllipR1, which covers most of the problematic sections of the main linac.

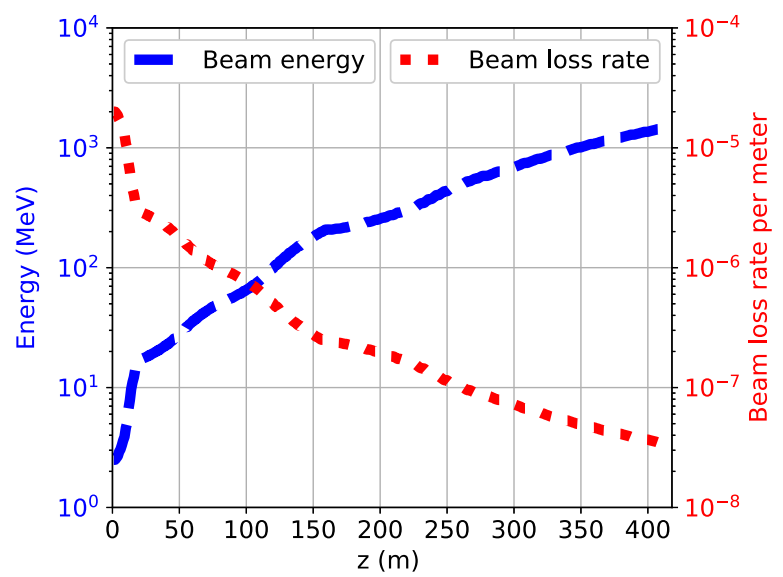

FIG. 13. Beam energy of the linac (blue dashed line) and $1 \mathrm{~W} / \mathrm{m}$ beam loss rate (red dotted line).
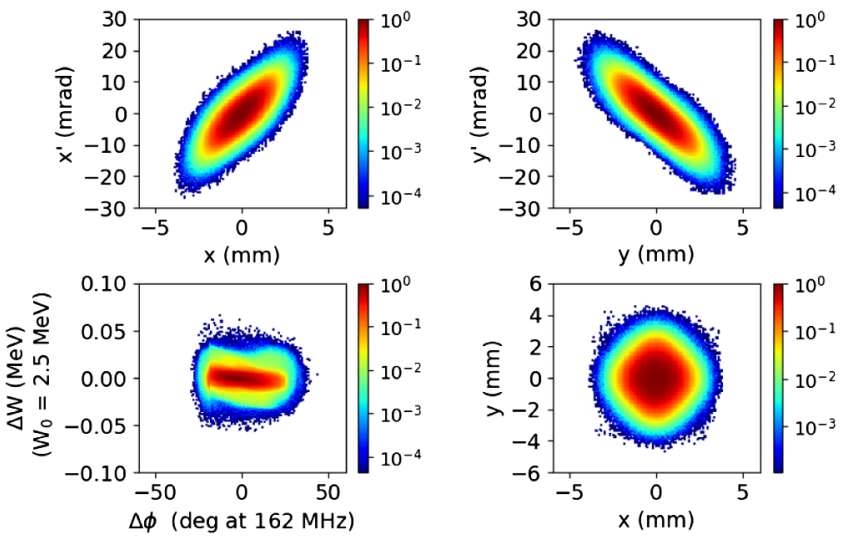

FIG. 14. Beam distribution at the entrance of the MEBT.

The particle distribution acquired from an early PARMTEQM [36] RFQ design [37] was used as an input for the simulation, as illustrated in Fig. 14. Table VI provides a summary of the simulation parameters. First, we investigated the beam core behavior by analyzing the evolution of rms beam parameters. Then, the beam halo was studied by the outermost fractions of the beam and emittance.

A close-up view of the Hofmann chart for the main linac provided in Sec. II is shown in Fig. 15. The working points are nearby the equipartitioning condition. Due to the difficulties of beam matching, the working points at the HWR, yellow dots, have the maximum dispersion around the equipartitioning requirement. Some of these spots are in the third and fourth resonance areas, although the rate of emittance exchange at those places is slow.

The evolution of the envelopes on the three planes is shown in Fig. 16. The acceptance to rms beam size ratios are also presented at the bottom of Fig. 16. The ratios of the linac aperture to the rms beam size are shown on the transverse plane. High-intensity linacs pursue a fraction of 10 [38], whereas our values vary from 10 to 20 . On the longitudinal, the typical $\left|\phi_{s}\right|$ to the rms phase width ratio is 10 [20]. However, we calculated the ratio of $2\left|\phi_{s}\right|$, the fullwidth acceptance's upper limit, to the rms phase size. Our design increased the computed fraction from 20 to 100 .

TABLE VI. Parameters for the particle simulation of the MEBT and main linac.

\begin{tabular}{lc}
\hline \hline Parameters & \\
\hline Length $(\mathrm{m})$ & 416 \\
Number of cavities & 295 \\
Number of magnets & 157 \\
Number of macroparticles & $1 \times 10^{7}$ \\
Input $\varepsilon_{\text {norm,rms,x }}(\pi \mathrm{mm} \mathrm{mrad})$ & 0.20 \\
Input $\varepsilon_{\text {norm,rms,y }}(\pi \mathrm{mm} \mathrm{mrad})$ & 0.21 \\
Input $\varepsilon_{\text {norm,rms, }}(\pi \mathrm{MeV} \mathrm{deg} / \mathrm{mm} \mathrm{mrad})$ & $0.07 / 0.37$ \\
\hline
\end{tabular}




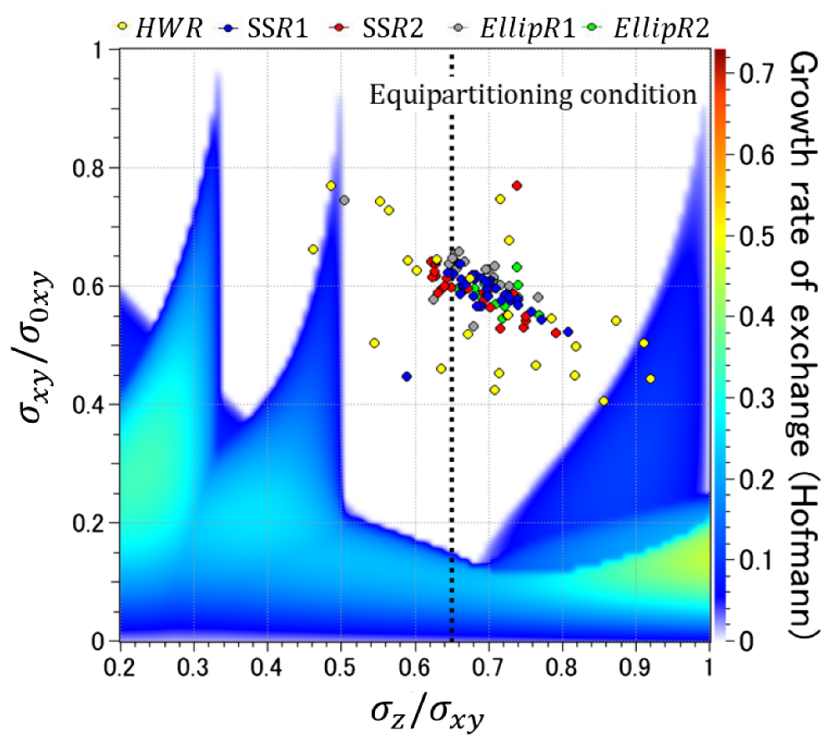

FIG. 15. Hofmann chart for the main linac.
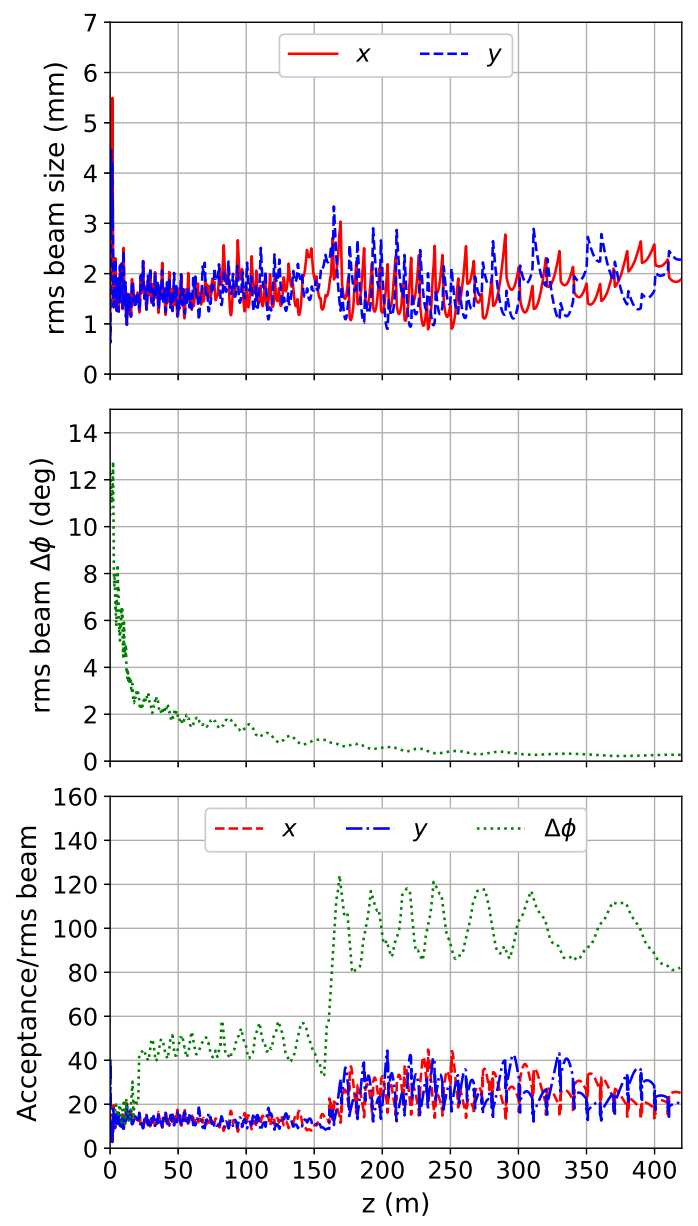

FIG. 16. Rms beam size along the MEBT and main linac. The upper plot shows the transverse rms envelopes. The middle plot is the rms phase width. Finally, the bottom one presents the ratio between the acceptance, aperture radius for the transverse plane and $2\left|\phi_{s}\right|$ for the longitudinal, and the beam rms size.

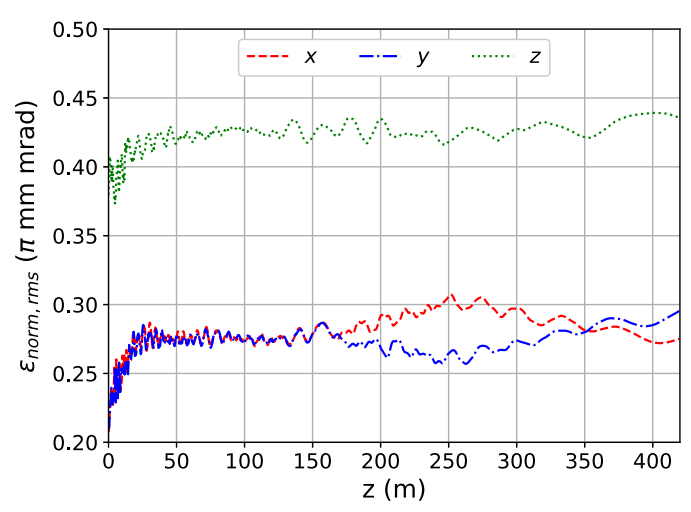

FIG. 17. Normalized rms emittance evolution along the MEBT and main linac.

This finding corresponds to a ratio of $\phi_{s}$ to the rms phase ranging from 10 to 50 .

Figure 17 shows the evolution of the $\varepsilon_{\text {norm,rms }}$ through the MEBT and the main linac. The emittance growth was computed as $\varepsilon / \varepsilon_{0}$ where $\varepsilon$ is the emittance at some point along the linac for a specific direction, and $\varepsilon_{0}$ is the initial emittance for that direction. The final emittance growths rates were $32.4 \%$ on $x$ axis, $39.2 \%$ on $y$ axis, and $14.7 \%$ on the $z$ axis. The rms envelopes and emittance demonstrated the stability of the beam core through the MEBT and main linac.

The next step was to investigate the behavior of the beam halo. We were able to model the evolution of particles up to six rms beam size out from the beam center using the $10^{7}$. The evolution of the beam halo was explored by examining the advancement of the various beam fractions along the linac, as shown in Fig. 18. The transverse aperture to outermost beam density fraction ratio was on average 3 . The phase acceptance value ranged from 2.5 to 7 in the low- $\beta$ cavity region to 15 in the high- $\beta$ cavity region.

In addition, Fig. 19 depicts the emittance growth at different fractions to investigate the beam halo formation. The evolution growths for the emittance fraction up to $99 \%$, three-rms beam size, were less than two on the transverse plane. The horizontal direction showed a growth that was less than seven for full emittance; on the contrary, the vertical direction showed a growth that was slightly above seven. In contrast, the longitudinal emittance growth was successfully controlled: all beam fractions showed a rise of less than two. Finally, beam scraping at the MEBT was required to avoid beam loss at the main linac. Especially in the HWR sector, where the beam degradation was the most severe. It is also important to mention that, even if the number of particles is increased, to achieve the ideal $10^{8}$ macroparticles for the beam lost evaluation EllipR2 section, the linac's aperture has enough room to contain them. Figure 20 shows the beam distribution at the end of the linac. This error-free model is referred to as the ideal machine. 


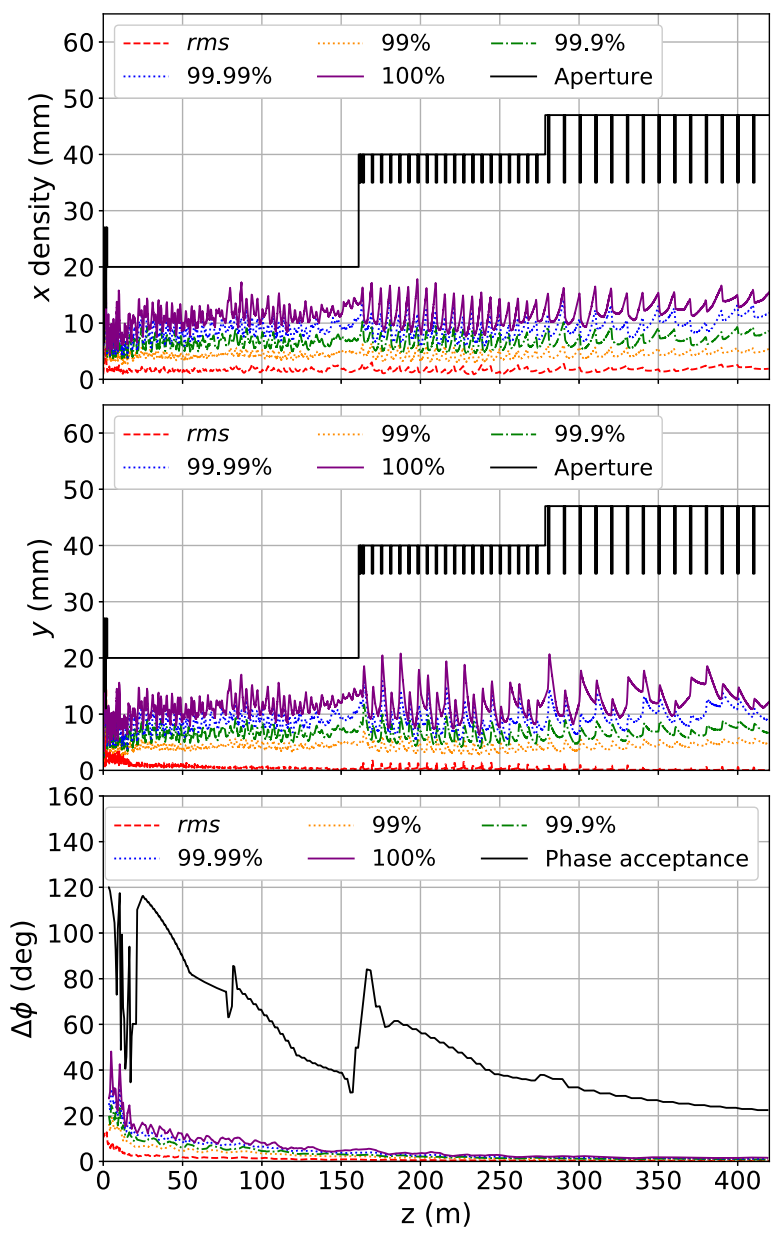

FIG. 18. Beam evolution along the linac for different beam fractions. The aperture line is the physical aperture of the linac elements and the phase acceptance one is the $2\left|\phi_{s}\right|$ of the cavities.

\section{B. Error studies}

A series of error studies were carried out as the next step toward the robustness lattice design to evaluate the linac tolerances for the optimal JAEA-ADS design. TraceWin was used to run simulations from the MEBT to the end of the main linac.

The simulated errors were divided into two categories: element errors (EE) and input beam errors (IBE). A summary of these errors is presented in Tables VII and VIII for EE and IBE, respectively. The amplitude errors were chosen based on the beam performance utilizing multiparticle simulations and error studies of high-intensity hadron linacs [20,28,39-41]. We will employ alignment strategies using optical systems [42], wire position monitor [43], and cryomodule assembly approaches [44] to attain those tolerances. We will use a digital low-level-rf control system [45] and field programmable gate array technology [46] to achieve phase and amplitude tolerances.

EE cases are usually classified as static or dynamic based on their duration time. Because static errors remain constant over long periods of time, they can be measured and
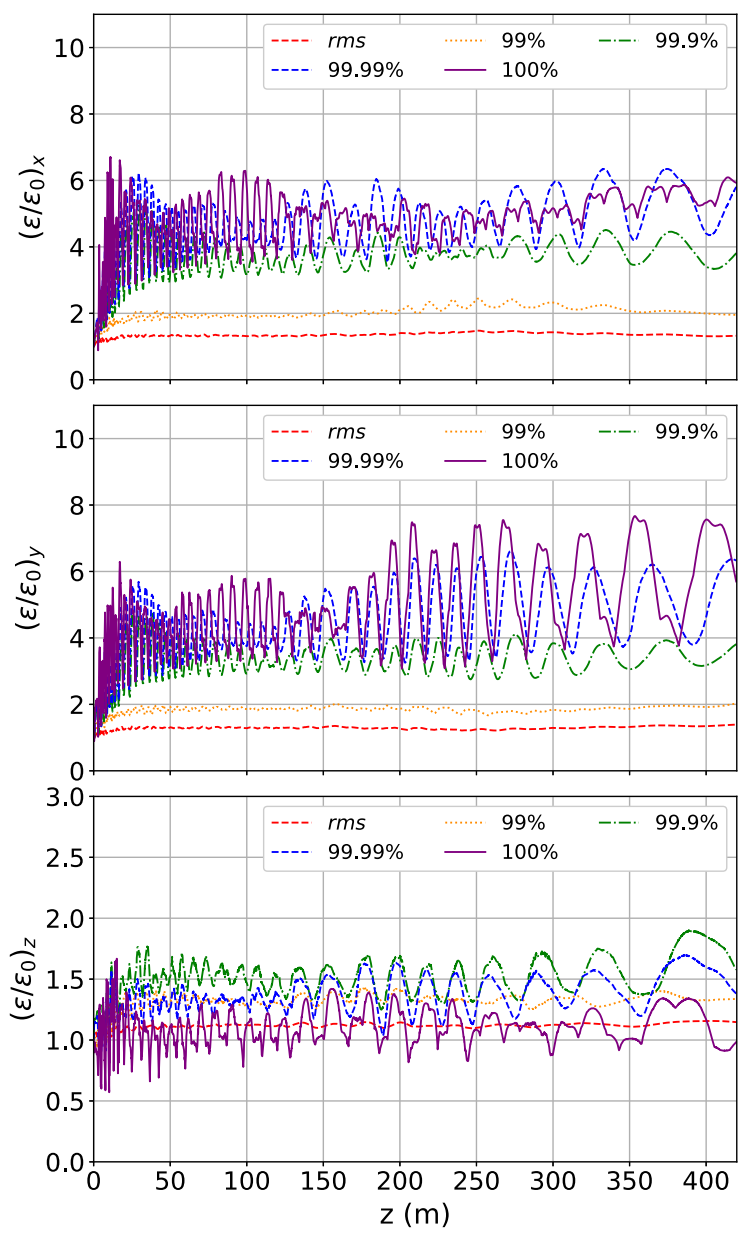

FIG. 19. Halo behavior through the linac for different beam emittance fractions.

compensated. Typically, static type occurs during machine installation or after a long period of operation. Dynamics errors are random and abrupt; therefore, they remain uncompensated.
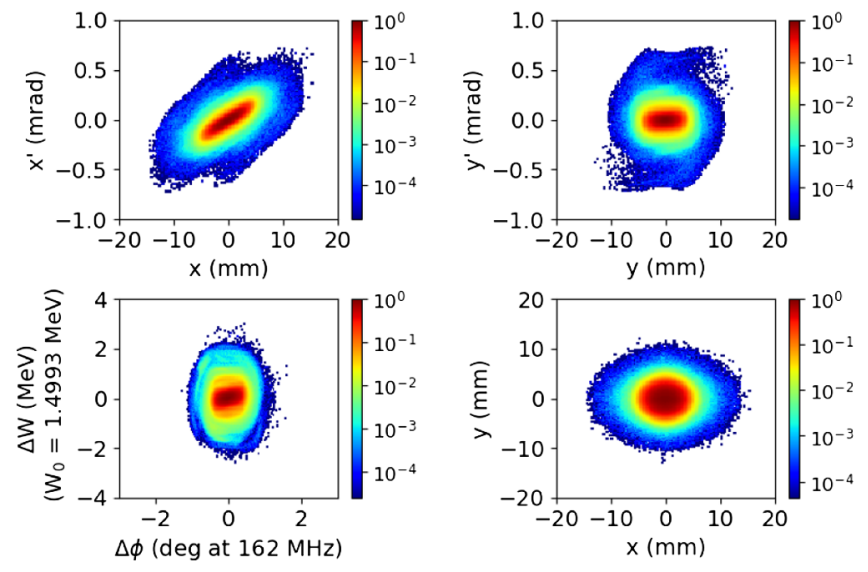

FIG. 20. Beam distribution at the end of the linac. 
TABLE VII. Summary of the element errors (EE) for the JAEA-ADS.

\begin{tabular}{|c|c|c|c|c|c|c|c|c|c|}
\hline \multirow[b]{2}{*}{ Error } & \multirow[b]{2}{*}{ Description } & \multicolumn{2}{|c|}{ MEBT } & \multicolumn{2}{|c|}{ HWR } & \multicolumn{2}{|c|}{ SSR } & \multicolumn{2}{|c|}{ EllipR } \\
\hline & & Static & Dynamic & Static & Dynamic & Static & Dynamic & Static & Dynamic \\
\hline$\overline{\mathrm{EE} 1}$ & Magnet $\Delta x, \Delta y^{\mathrm{a}}(\mathrm{mm})$ & 0.45 & 0.005 & 0.85 & 0.009 & 0.85 & 0.009 & 0.45 & 0.005 \\
\hline EE2 & Magnet $\Delta \theta x, \Delta \theta_{y}^{\mathrm{b}}(\mathrm{mrad})$ & 0 & 0 & 2 & 0.02 & 2 & 0.02 & 0 & 0 \\
\hline EE3 & Magnet $\Delta \theta_{z}(\mathrm{mrad})$ & 5 & 0.05 & 0 & 0 & 0 & 0 & 5 & 0.05 \\
\hline EE4 & Magnet gradient $(\%)$ & 0.5 & 0.1 & 0.5 & 0.1 & 0.5 & 0.1 & 0.5 & 0.1 \\
\hline EE5 & Cavity $\Delta x, \Delta y(\mathrm{~mm})$ & 0.4 & 0.004 & 0.85 & 0.009 & 0.85 & 0.009 & 0.85 & 0.009 \\
\hline EE6 & Cavity $\Delta \theta_{x}, \Delta \theta_{y}(\mathrm{mrad})$ & 3.5 & 0.035 & 6 & 0.06 & 6 & 0.06 & 3.5 & 0.035 \\
\hline EE7 & Cavity rf phase (deg) & 1 & 0.5 & 1 & 0.5 & 1 & 0.5 & 1 & 0.5 \\
\hline EE8 & Cavity rf amplitude (\%) & 1 & 0.5 & 1 & 0.5 & 1 & 0.5 & 1 & 0.5 \\
\hline
\end{tabular}

${ }^{\mathrm{a}} \Delta x$ and $\Delta y$ are displacement in the horizontal and vertical direction, respectively.

${ }^{\mathrm{b}}$ The error of $\Delta \theta_{u}$ indicates rotation error around the subscript axis of the quadrupole magnets.

Dynamic misalignments of elements, attribute mainly by ground motion, are described by the ATL-Law [47]:

$$
d R^{2} \approx \mathrm{ATL}
$$

where $A$ is of the order of $10^{-5 \pm 1}\left(\frac{\mu \mathrm{m}^{2}}{\mathrm{~s}}\right) / \mathrm{m}, T$ is the spatial time scale, and $L$ is the spatial length. Typically, the values utilized are a few tenths of a micron that correspond to a small percentage of statics values. On the contrary, $\mathrm{rf}$ and magnetic fields dynamics values represent a fraction of static error amplitude. The IBE indicates the error effects of the first part of the linac, from the ion source to the RFQ, and are fluctuations of the initial beam parameters at the MEBT.

Error experiments were carried out in two stages. First, we used the so-called single error simulation to apply each of the faults separately. Second, we incorporated all of the same types of EE or IBE errors at the same time, a process known as combined errors.

Each error study consisted of 1000 independent runs with a beam distribution of $1 \times 10^{5}$ macroparticles, for a cumulative statistics of $10^{8}$ macroparticles. This proceeding is similar to the one applied for other projects $[20,39,41]$. We use that configuration because it was the best in terms of computational time and because $1 \times 10^{5}$ macroparticles allow us to predict beam losses in the most troublesome locations, the HWR and the beginning of SSR1. As previously stated, the final error tolerances were chosen as a compromise between the performance of other high-intensity linacs and keeping the beam loss in our linac below $1 \mathrm{~W} / \mathrm{m}$.

To that purpose, we calculated the following figures of merit: (1) Beam loss, (2) Final normalized rms-emittance ratio defined as $\varepsilon_{e} / \varepsilon_{i}$ between the error case $\varepsilon_{e}$ and the ideal machine case $\varepsilon_{i}$, and (3) The evolution of the beam centroid by computing the rms residual orbit error

$$
R_{\mathrm{rms}, \mathrm{roe}}=\sqrt{\frac{1}{N} \sum_{j=1}^{N} R_{j}^{2}-\bar{R}},
$$

where

$$
\bar{R}=\frac{1}{N} \sum_{j=1}^{N} R_{j},
$$

$R$ is the beam gravity position in one of the three axes and $N$ is the number of simulation runs; (4) The maximum and rms beam size at the end of the linac.

We started with the single static errors of the EE group. Table VII presents a summary of error amplitude for the static and dynamic EE cases. Because of the axis symmetry, only the errors on the horizontal direction were simulated for the position and rotation errors on the transverse plane. For single EE errors, the error amplitude was increased in five steps from zero to its maximum value. The error amplitude was uniformly distributed within plus-minus the maximum available error value in each step. Figure 21

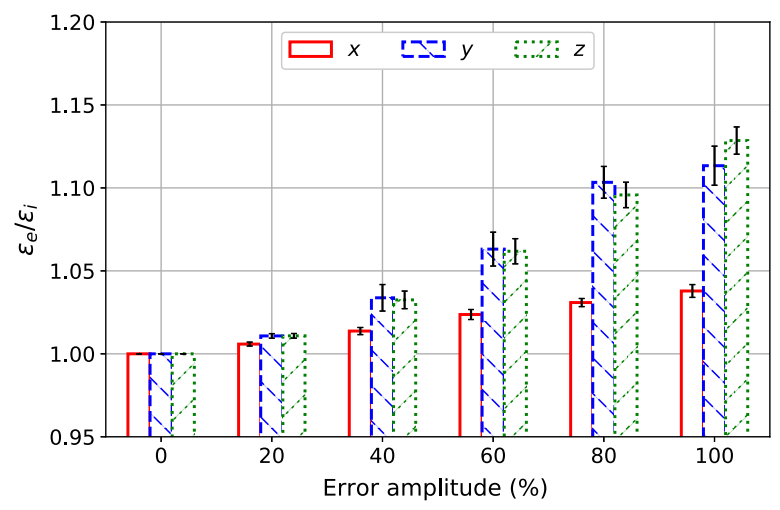

FIG. 21. Error amplitude scan of the emittance growth for the static EE2 case. Error bars represent the standard deviation error of the mean of 1000 runs. 


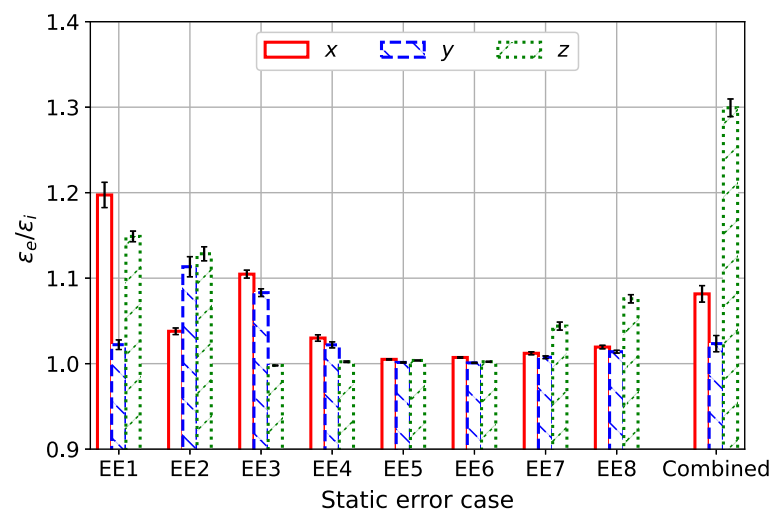

FIG. 22. Summary of the average emittance growth for the different static EE defined in Table VII.

shows the emittance growth at the end of the linac as a function of the error amplitude percentage for the static EE2 case, rotation error on the transverse plane in magnets. The plot reveals a considerable increase when the error value exceeds $60 \%$ of the maximum error amplitude.

Figure 22 presents a summary of the emittance growths for single static EE cases to the ideal machine. Additionally, the combined case described below is also shown. Misalignment errors provided the greatest emittance rise for single errors; nevertheless, only EE1 and EE2 detected beam loss. These two cases produced a large transverse beam centroid offset that made the envelope hit the linac aperture. EE2 had the most beam losses of all of them. The reason is that the transverse rotation error has a significant effect on the transverse focusing in the solenoid regions,
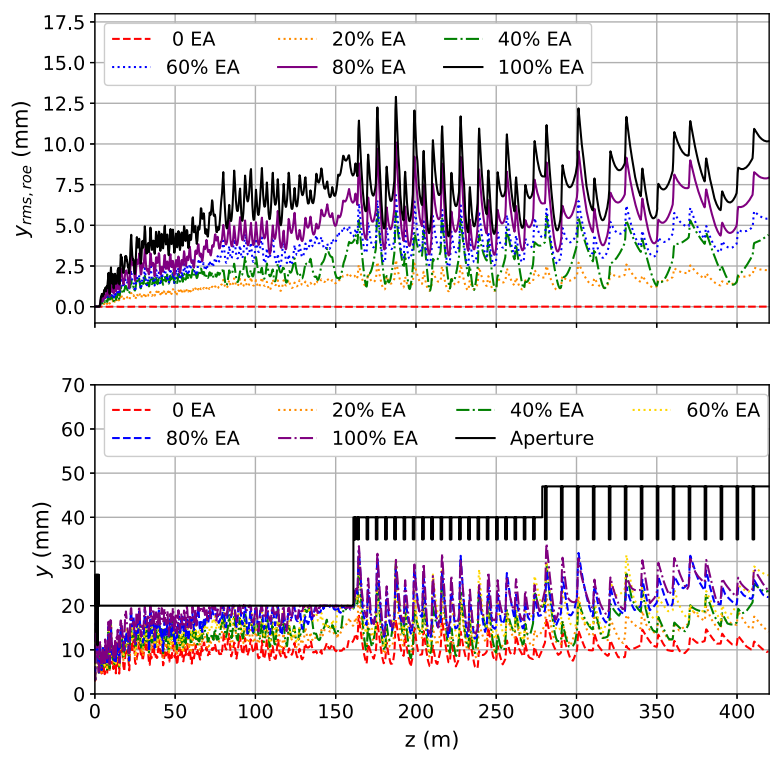

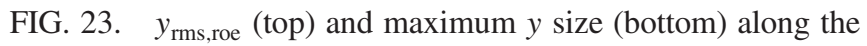
linac for the different error amplitude (EA) cases.
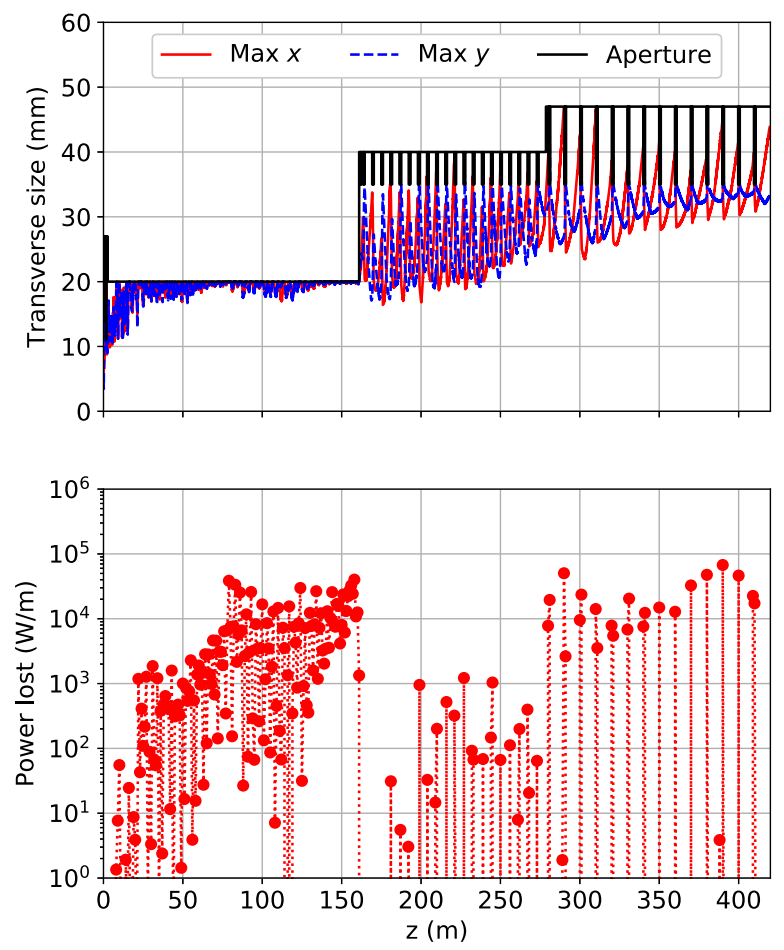

FIG. 24. Maximum transverse size (top) and beam power lost per meter (bottom) along the linac when all the static EE cases were applied simultaneously with the error value uniformly distributed within plus-minus their amplitude.

where the phase advance and space-charge are large. Thus, the rotation error around the horizontal axis $\theta_{x}$ causes strong orbit distortion in the $y$ direction. Figure 23 shows the vertical rms residual orbit error (top) and the maximum beam size (bottom) along the linac for different error amplitude percentages for the EE2. The $y_{\text {rms,roe }}$ is significantly increased in the HWR section, resulting in a large beam size in the following sections. The vertical envelope reached the aperture at the SSR2 from an error amplitude of $40 \%$. At $100 \%$, the envelope in the $y$ direction impacted the aperture in almost all of the SSR sections, resulting in severe beam losses.

Figure 24 depicts the maximum transverse envelopes (top) and their corresponding power lost per meter (bottom) for the combined error case, where the errors are equally distributed plus-minus the maximum error amplitude. On the contrary to the single static error cases, the errors were applied in all the axes for the combined errors. The envelopes reached the aperture at the last cryomodule of the HWR section, resulting in a significant loss of power. $65 \%$ of the 1000 cases had losses above $1 \mathrm{~W} / \mathrm{m}$, primarily in the low- $\beta$ section. These findings restrict the maximum alignment tolerance of the elements.

For the static case, the error amplitudes proposed in Table VII produced a large transverse offset that results in 
(a)
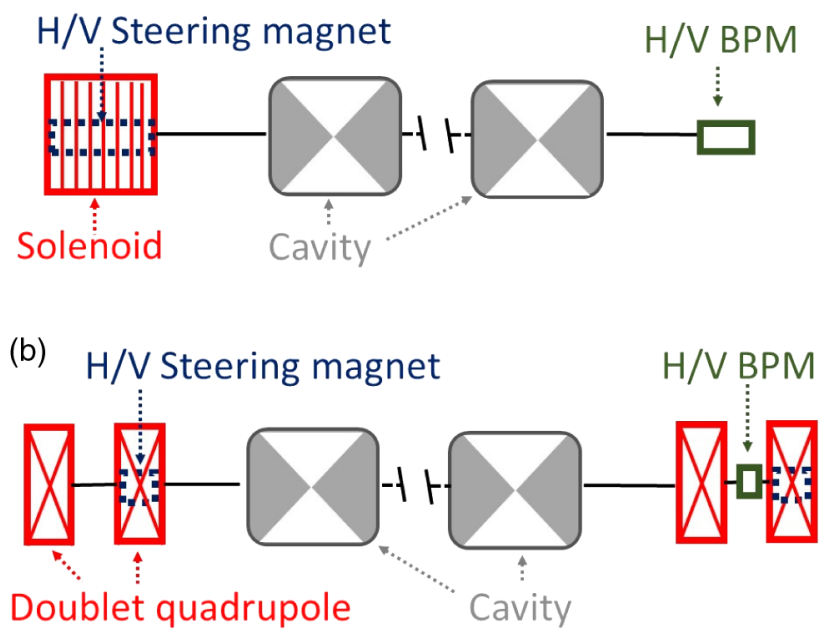

\section{$\mathrm{H}=$ Horizontal, $\mathrm{V}=$ Vertical, $\mathrm{BPM}=$ Beam position monitor}

FIG. 25. Orbit correction schemes implemented for the JAEAADS linac. The scheme (a) is implemented for the HWR and SSRs sections, and the (b), for the elliptical ones.

beam loss, as is shown in Figs. 24 and 26. Beam-based alignment methods can restore the beam centroid offset since these inaccuracies remain constant over lengthy periods of time. One-to-one steering [48] was chosen because of its simplicity and widespread use during beam commissioning around the world. Figure 25 presents the
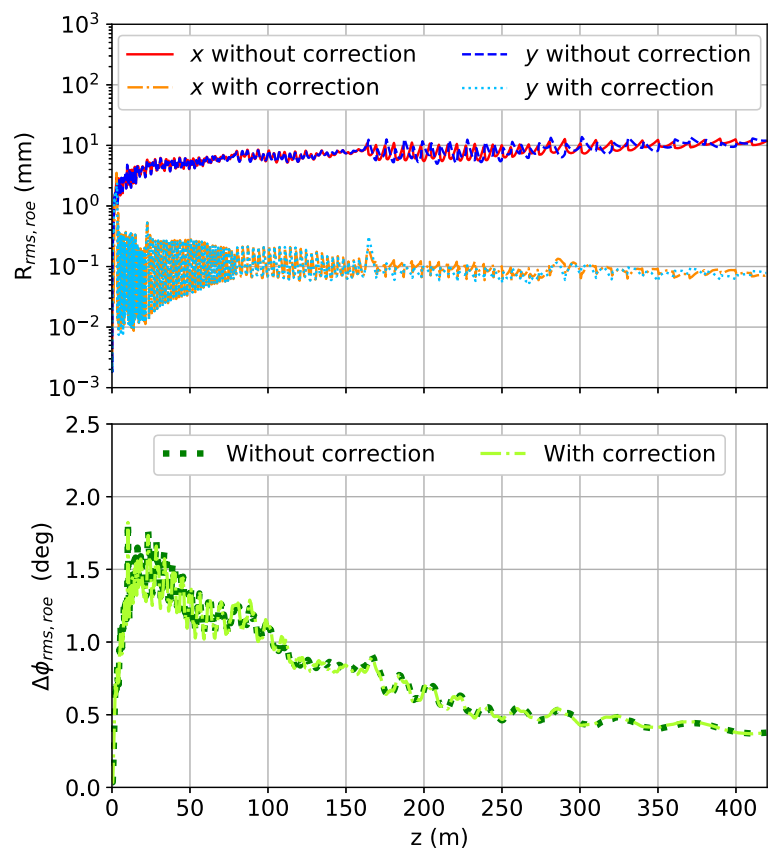

FIG. 26. Transverse (top) and longitudinal (bottom) rms of residual orbit error for the cases without and with orbit correction when all the static EE are combined.

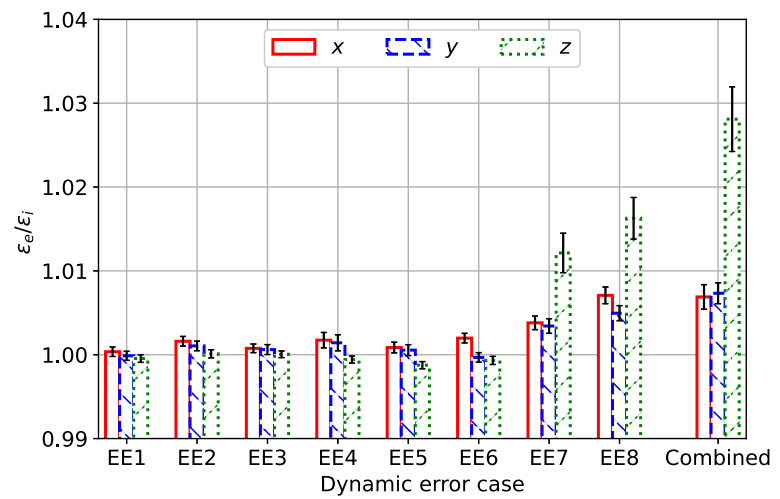

FIG. 27. Summary of the average emittance growth for the different dynamic EE defined in Table VII.

orbit correction schemes for the JAEA-ADS linac. At lowbeta, we employ combined focusing solenoids and steering coils associated with BPM to keep a compact period [49,50], especially at HWR sections. We used a steering configuration based on SPS research [39] in the elliptical cavities. During the orbit correction studies, the BPMs were simulated with an accuracy of $100 \mu \mathrm{m}$ [51] to account for their displacements and measurement resolution.

The effectiveness of the correction scheme was demonstrated by applying to the combined element errors. The orbit correction in the simulation was conducted using standard diagnostic functions of TraceWin. Figure 26 shows the largest rms residual orbit error on the transverse direction is above $10 \mathrm{~mm}$ and $1.8 \mathrm{deg}$ on the longitudinal one. The orbit correction reduces the transverse residual orbit error along the main linac to $0.3 \mathrm{~mm}$. The maximum steering strength in the corrector was less than $5 \times 10^{-3} \mathrm{Tm}$.

The largest beam envelopes for all error situations correspond to a cumulative density of $10^{8}$ macroparticles, as shown in Fig. 29. In addition, as a point of reference, the ideal machine was presented. At the last HWR cryomodule, the transverse envelopes for the case without correction scheme, the yellow dotted line, reached the aperture.

TABLE VIII. Summary of the input beam errors (IBE).

\begin{tabular}{llc}
\hline \hline Error & Description & Error amplitude \\
\hline IBE1 & $\Delta x, \Delta y(\mathrm{~mm})$ & 1 \\
IBE2 & $\Delta \phi(\mathrm{deg})$ & 1 \\
IBE3 & $\Delta x^{\prime}, \Delta y^{\prime}(\mathrm{mrad})$ & 1 \\
IBE4 & Energy $(\mathrm{keV})$ & 12.5 \\
IBE5 & $\varepsilon_{x}, \varepsilon_{y}$ growth $(\%)$ & 10 \\
IBE6 & $\varepsilon_{z}$ growth $(\%)^{\text {growatch }}$, Mismatch $_{y}(\%)$ & 10 \\
IBE7 & Mismatch $_{x}(\%)$ & 5 \\
IBE8 & Mismatch $_{z}(\%)$ & 5 \\
IBE9 & $\Delta I_{\text {beam }}(\mathrm{mA})$ & 0.2 \\
\hline \hline
\end{tabular}




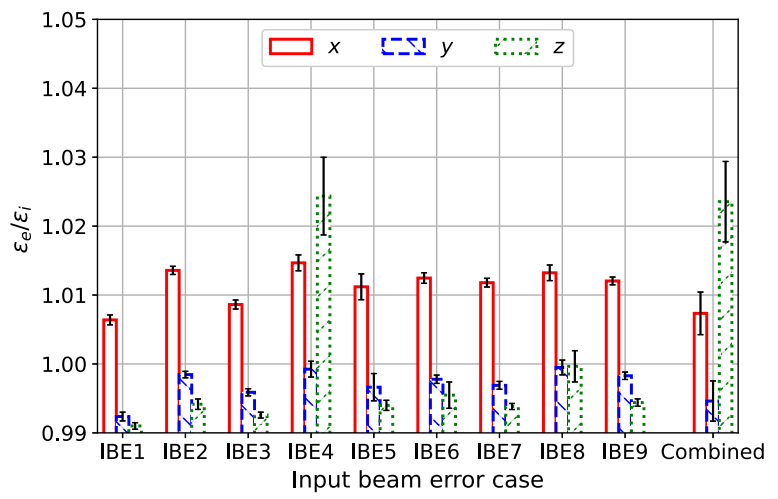

FIG. 28. Emittance growth of the input beam errors with respect to the ideal machine at the exit of the linac. The error bars are the standard error of the mean.

The envelopes for the correction scheme, the green dasheddotted line, on the other hand, recovered behavior similar to the ideal machine, the red dashed line. As a result of the correction technique, we recorded beam loss of the order of $10^{-2} \mathrm{~W} / \mathrm{m}$ at the entrance of the first HWR cryomodule. The final average energy was $1499.35 \mathrm{MeV}$ with an rms of $0.34 \mathrm{MeV}$.

The dynamic EE cases were then analyzed using the same procedure as the static cases, with the exception that the correction scheme was not used in these studies. The emittance growths induced by each of the errors separately and combined are illustrated in Fig. 27 for the tolerance stated in Table VII. In all cases, emittance growth was less than $3 \%$, nonbeam loss was recorded, and the rest of the figure of merit was nearly identical to the ideal machine case.

The input beam errors were the last error cases to be simulated. The error investigations were conducted in the same manner as the element errors. Table VIII describes the errors and their amplitudes.

Figure 28 shows a summary of the emittance growth for the perfect machine situation, similar to Figs. 22 and 27. The biggest increase in emittance was caused by a single energy error; nonetheless, the values were less than $3 \%$. In the combined case, the increase in emittance was also less than $3 \%$. The input beam error has a minimal impact on the beam performance in dynamic EE cases.

Finally, Table IX summarizes the beam parameters used to quantify the error's impact on lattice performance for both the ideal and error cases. Unwanted beam losses did not occur in the ideal machine, and the final normalized rms emittance growth was up to $40 \%$. Additionally, the envelopes are well contained in the apertures and phase acceptance. In contrast, for the static element errors, the beam reached the aperture at the low- $\beta$ sections. Consequently, the beam loss rate exceeded the safety limits of beam operation. This problem was overcome by implementing a correction scheme that corrected the transverse beam offset and brought the accelerator's performance closer to the ideal machine. The dynamic element and input beam errors had a negligible impact on the beam performance. Figure 29 presents the maximum envelopes for the ideal machine and error cases. All the envelopes for the error cases have almost the same amplitude, except the static error element without compensation. As a result, it emphasizes the importance of the correction scheme

TABLE IX. Summary of beam parameters for the different error cases at the end of the linac.

\begin{tabular}{lccccc}
\hline \hline & & \multicolumn{3}{c}{ Element error } & \\
\cline { 3 - 4 } & & \multicolumn{2}{c}{ Static } & \\
\cline { 3 - 4 } Parameters & Ideal machine & Without correction & With correction & Dynamic & Input beam error \\
\hline Total proton lost ${ }^{\mathrm{a}}(\%)$ & 0 & 51 & $3.5 \times 10^{-3}$ & 0 & 0 \\
Max proton lost rate $(1 / \mathrm{m})$ & 0 & $3.9 \times 10^{-2}$ & $3.6 \times 10^{-7}$ & 0 & 0 \\
Max beam power lost $(\mathrm{W} / \mathrm{m})$ & 0 & $6.7 \times 10^{4}$ & $1.8 \times 10^{-2}$ & 0 & 0 \\
$\left(\varepsilon / \varepsilon_{0}{ }^{\mathrm{b}}\right) x$ & 1.32 & 1.43 & 1.54 & 33.8 & 33.2 \\
$\left(\varepsilon / \varepsilon_{0}\right) y$ & 1.39 & 1.42 & 1.55 & 1.40 & 1.38 \\
$\left(\varepsilon / \varepsilon_{0}\right) z$ & 1.14 & 1.49 & 1.29 & 1.18 & 1.17 \\
$\mathrm{X}_{\text {rms,roe }}(\mathrm{mm})$ & $3.4 \times 10^{-4}$ & 11.78 & 0.07 & 0.15 & $7.9 \times 10^{-3}$ \\
$\mathrm{y}_{\text {rms,roe }}(\mathrm{mm})$ & $3.3 \times 10^{-4}$ & 11.98 & 0.08 & 0.16 & $9.6 \times 10^{-3}$ \\
$\Delta \phi_{\text {rms,roe }}(\mathrm{deg})$ & 0.02 & 0.39 & 0.38 & 0.19 & 0.08 \\
Envelope $\mathrm{x}(\mathrm{Max} / \mathrm{rms})(\mathrm{mm})$ & $15.5 / 1.9$ & $43.8 / 1.9$ & $17.9 / 2.3$ & $15.9 / 2.2$ & $16.1 / 2.2$ \\
Envelope $\mathrm{y}($ Max/rms) $(\mathrm{mm})$ & $12.2 / 2.3$ & $33.3 / 1.8$ & $13.9 / 1.9$ & $13.7 / 1.9$ & $12.4 / 1.9$ \\
Envelope $\Delta \phi(\mathrm{Max} / \mathrm{rms})(\mathrm{deg})$ & $1.6 / 0.3$ & $2.5 / 0.2$ & $2.5 / 0.3$ & $2.0 / 0.2$ & $1.7 / 0.2$ \\
\hline \hline
\end{tabular}

${ }^{\mathrm{a}}$ The scraped protons at the collimator are excluded.

${ }^{\mathrm{b}} \varepsilon_{0}$ is the emittance at the entrance of the MEBT section. 



FIG. 29. Maximum transverse and phase envelopes for the ideal machine and error cases. The envelopes plotted correspond to a cumulative statistic of $10^{8}$ macroparticles.

operating with error tolerance comparable to the state-ofthe-art in high-intensity linacs.

\section{CONCLUSIONS}

Almost two decades have passed since JAERI, now JAEA, designed the first ADS linac. The current JAEAADS linac operates with a beam current of $20 \mathrm{~mA}$ and final energy of $1.5 \mathrm{GeV}$; consequently, it is a $30-\mathrm{MW}$ proton linac, the highest-beam power ADS linac to date. The present linac implemented the most recent advancements in superconducting cavities and used an optimization approach to achieve high acceleration efficiency and reliability. As a result, the numbers of different rf structures and the linac length were reduced to half compared to the previous design, NSRP.

SC cavities replace the NC section of the NSRP linac. Due to the decreased density of the focusing components, the low-beta part of the SC linac is dominated by spacecharge even with an intermediate current of $20 \mathrm{~mA}$, in contrast to NC linacs that employ rf structures such as DTLs. By selecting appropriate parameters to control the space-charge effect, we were able to create a robust lattice design even under the space-charge dominant condition. The linac optics were optimized using an error-free case with a beam distribution of $10^{7}$ macroparticles. Beam losses were not recorded due to the beam scraping at the MEBT, and the rms emittance growth was lower than $40 \%$ on the transverse direction and $15 \%$ on the longitudinal direction.

The error cases allowed us to test the lattice design in more realistic conditions. Static element errors showed that misalignments in magnets were the principal source of transverse beam centroid offset that causes beam losses. Consequently, they are the greatest threat to linac stability. Multiple errors are expected during beam operation. In that situation, our simulations revealed that $65 \%$ of the cases recorded beam losses exceeding the limit of hands-on maintenance when no corrective scheme was applied. The one-to-one correction scheme was shown to be capable 
of restoring the transverse beam centroid and, subsequently, reducing beam losses by two orders of magnitude below $1 \mathrm{~W} / \mathrm{m}$. It also helped to control the emittance growth and beam envelopes. Therefore, a correction scheme for the JAEA-ADS linac is mandatory. The dynamic error element and input beam errors had a negligible effect on the linac performance. This study tested the robustness of the linac lattice to control the beam loss and the emittance growth with feasible error tolerance. Thus, it represents a step toward developing a reliability-oriented linac for the JAEAADS project.

\section{ACKNOWLEDGMENTS}

The authors would like to thank Professor Robert A. Jameson, Dr. Cristhian Valerio Lizarraga, Dr. Daniel Chavez, the members of the JAEA-ADS, J-PARC linac, KEK SRF, and Riken SRF group for their comments and suggestions. This work was financially supported by the Ministry of Education, Culture, Sports, Science and Technology (MEXT) as the Subsidy for Research and Development on Nuclear Transmutation Technology through the Japan Atomic Energy Agency (JAEA).

[1] T. Mukaiyama, H. Takano, T. Ogawa, T. Takizuka, and M. Mizumoto, Partitioning and transmutation studies at JAERI both under OMEGA program and High-Intensity Proton Accelerator project, Prog. Nucl. Energy 40, 403 (2002).

[2] D. Vandeplassche and L. Medeiros-Romão, Accelerator driven systems, in Proceedings of 3rd International Particle Accelerator Conference, New Orleans, U.S.A, 2012 (JACoW, Geneva, 2012), pp. 6-10.

[3] H. Takei, K. Nishihara, K. Tsujimoto, and H. Oigawa, Estimation of acceptable beam-trip frequencies of accelerators for accelerator-driven systems and comparison with existing performance data, J. Nucl. Sci. Technol. 49, 384 (2012).

[4] H. Takei, K. Furukawa, Y. Yano, and Y. Ogawa, Evaluation of mean time between accidental interruptions for accelerator klystron systems based on the reliability engineering method, J. Nucl. Sci. Technol. 55, 996 (2018).

[5] T. Sugawara, H. Takei, and K. Tsujimoto, Investigations of accelerator reliability and decay heat removal for accelerator-driven system, Ann. Nucl. Energy 125, 242 (2019).

[6] J.-L. Biarrotte, Technical Report No. CERN 2013-001481. (European Organization for Nuclear Research, Geneva, 2011).

[7] M. Mizumoto, K.Hasegawa, H. Yoliobori, H. Oguri, Y. Okumura, J. Kusano, H. Murata, K. Sakogawa, M. Kawai, T. Nishida, T.Takizuka, and H. Takada, Development of proton linear accelerator and transmutation system, in Proceedings of GLOBAL 1993, Seattle, U.S.A., 1993 (American Nuclear Society, 1993), pp. 357-362.

[8] M. Mizumoto, J. Kusano, K. Hasegawa, N. Ito, H. Oguri, Y. Touchi, K. Mukugi, and H. Ino, A high intensity proton linac development for Neutron Science Research Program, in Proceedings of the 18th International Linear Accelerator Conference, Geneva, Switzerland, 1996 (JACoW, Geneva, 1996), pp. 662-664.

[9] M. Mizumoto, J. Kusano, K. Hasegawa, N. Ouchi, H. Oguri, M. Kinsho, E. Chishiro, T. Tomisawa, Y. Touchi, M. Ikegami, Y. Honda, K. Mukugi, H. Ino, F. Noda, N. Akaoka, and H. Kaneko, A high intensity proton linac development for the JAERI Neutron Science Project, in Proceedings of the 19th International Linear Accelerator Conference, Geneva, Switzerland, 1998 (JACoW, Geneva, 1998), pp. 349-353.

[10] M. Kihara, KEK Report No. 86-106 (National Laboratory for High Energy Physics, Tsukuba, 1987).

[11] M. Furusaka et al., KEK-Reports No. 99-4; JAERI-Tech99-056 (National Laboratory for High Energy Physics and Japan Atomic Energy Research Institute, Tsukuba, 1999).

[12] J. R. Delayen, Medium- $\beta$ superconducting accelerating structures, in Proceedings of the 10th Workshop on RF Superconductivity Conference, Tsukuba, Japan, 2001 (KEK, Tsukuba, 2001), pp. 152-161.

[13] A. Facco, Tutorial on low beta cavity, in Proceedings of the 12th Workshop on RF Superconductivity Conference, New York, U.S.A., 2005 (Cornell University, Ithaca, 2005), pp. 21-33.

[14] B. Yee-Rendon, Y. Kondo, F. Maekawa, S. Meigo, and J. Tamura, Electromagnetic design of the low beta cavities for the JAEA ADS, J. Phys. Conf. Ser. 1350, 012197 (2019); Design of the elliptical superconducting cavities for the JAEA ADS, J. Phys. Conf. Ser. 1350, 012198 (2019); Beam optics design of the superconducting region of the JAEA ADS, J. Phys. Conf. Ser. 1350, 012120 (2019).

[15] B. Yee-Rendon, J. Tamura, Y. Kondo, K. Hasegawa, F. Maekawa, S. Meigo, and H. Oguri, Cavity and optics design of the accelerator for the JAEA-ADS project, in Proceedings of 16th Annual Meeting of Particle Accelerator Society of Japan, Kyoto, Japan, 2019 (PASJ, Kyoto, 2019), pp. 107-111.

[16] B. Yee-Rendon, J. Tamura, Y. Kondo, K. Hasegawa, F. Maekawa, S. Meigo, and H. Oguri, Present status of the R $\&$ D of the superconducting linac for the JAEA-ADS, J. Phys. Soc. Jpn. Conf. Proc. 33, 011043 (2021).

[17] B. Yee-Rendon, J. Tamura, Y. Kondo, F. Maekawa, S. Meigo, and H. Oguri, Error studies for the JAEA-ADS linac, in Proceedings of 17th Annual Meeting of Particle Accelerator Society of Japan, Matsuyama (online), Japan, 2020 (PASJ, Matsuyama, 2020), pp. 33-37.

[18] K. Tsujimoto, T. Sasa, K. Nishihara, H. Oigawa, and H. Takano, Neutronics design for lead-bismuth cooled accelerator-driven system for transmutation of minor actinide, J. Nucl. Sci. Technol. 41, 21 (2004).

[19] D. Vandeplassche, J. L. Biarrote, H. Klein, and H. Podlech, The MYRRHA linear accelerator, in Proceedings of 2 nd International Particle Accelerator Conference, San Sebastian, Spain, 2011 (JACoW, Geneva, 2011), pp. 2718-2720.

[20] Z. Li, P. Cheng, H. Geng, Z. Guo, Y. He, C. Meng, H. Ouyang, S. Pei, B. Sun, J. Sun, J. Tang, F. Yan, Y. Yang, C. Zhang, and Z. Yang, Physics design of an accelerator for an accelerator-driven subcritical system, Phys. Rev. ST Accel. Beams 16, 080101 (2013). 
[21] I. Hofmann, L. Laslett, and I. Haber, Stability of the Kapchinskij-Vladimirskij (K-V) distribution in long periodic transport systems, Part. Accel. 13, 145 (1983), http:// cds.cern.ch/record/140869/files/p145.pdf.

[22] I. Hofmann, Stability of anisotropic beams with space charge, Phys. Rev. E 57, 4713 (1998).

[23] R. A. Jameson, Beam-intensity limitations in linear accelerators, IEEE Trans. Nucl. Sci. 28, 2408 (1981).

[24] B. Yee-Rendon, Y. Kondo, F. Maekawa, S. Meigo, and J. Tamura, Design of the MEBT for the JAEA-ADS project, in Proceedings of the 12th International Particle Accelerator Conference, Campinas, Brazil, 2021 (JACoW, Campinas, 2021), pp. 790-793.

[25] D. Uriot and N. Pichoff, TraceWin Manual, CEA Saclay (CEA/SACLAY-DRF/Irfu/DACM, 2020).

[26] P. A. P. Nghiem, B. Dalena, J. Dumas, N. Pichoff, and D. Uriot, Strategy of beam tuning implementation for the SARAF MEBT and SC linac, in Proceedings of the 8th International Particle Accelerator Conference, Copenhagen, Denmark, 2017 (JACoW, Geneva, 2017), pp. 652-654.

[27] T. P. Wangler, RF Linear Accelerators (WILEY-VCH Verlag GmbH \& Co. KGaA, Weinheim, 2008).

[28] M. Ball et al., The PIP-II Conceptual Design Report No. FERMILAB-DESIGN- 2017-01 (Fermi National Accelerator Laboratory, Batavia, 2017).

[29] Computer simulation technology, https://www.cst.com/.

[30] K. Halbach and R. F. Holsinger, SUPERFISH-a computer program for evaluation of rf cavities with cylindrical symmetry, Part. Accel. 7, 213 (1976), http://cds.cern.ch/ record/700607/files/p213.pdf.

[31] D. Uriot, GenLinWin Manual, CEA Saclay (CEA/SACLAYDRF/Irfu/DACM, 2014).

[32] P. N. Ostroumov, Physics design of $8 \mathrm{GeV} \mathrm{H}$-minus linac, New J. Phys. 8, 281 (2006).

[33] R. Duperrier, N. Pichoff, and D. Uriot, Frequency jump in an ion linac, Phys. Rev. ST Accel. Beams 10, 084201 (2007).

[34] P. N. Ostroumov, S. H. Kim, E. S. Lessner, K. W. Shepard, R. E. Laxdal, and R. Wheatley, A new generation of superconducting solenoids for heavy-ion linac application, in Proceedings of 21st International Linear Accelerator Conference, Gyeongju, Korea, 2002 (Pohang Accelerator Laboratory, Pohang, 2002), pp. 332-334.

[35] J.-L.Biarrotte, D.Uriot, F. Bouly, J.-P. Carneiro, and D. Vandeplassche, Design of the MYRRHA 17-600 MeV Superconducting LINAC, in Proceedings of the 16th International Conference of RF Superconductivity, Paris, France, 2013 (JACoW, Geneva, 2013), pp. 129-132.

[36] K. R. Crandall et al., RFQ Design Codes, Report No. LA-UR- 96-1836 (Los Alamos National Laboratory, Los Alamos, 2005).

[37] Y. Kondo, J. Tamura, and B. Yee-Rendon, Reference design of the RFQ for JAEA ADS linac, J. Phys. Soc. Jpn. Conf. Proc. 33, 011015 (2021).
[38] R. A. Jameson, On scaling \& optimization of highintensity, low-beam-loss RF linacs for neutron source drivers, AIP Conf. Proc. 279, 969 (1992).

[39] F. Gerigk et al., Conceptual design of the SPL II, CERN Report No. 2006-006 (European Organization for Nuclear Research, Geneva, 2006).

[40] P. N. Ostroumov, V. N. Aseev, and B. Mustapha, Beam loss studies in high-intensities heavy-ions linacs, Phys. Rev. ST Accel. Beams 7, 090101 (2004).

[41] R. Garoby et al., The European spallation source design, Phys. Scr. 93, 014001 (2018).

[42] J. Gayde, Y. Kadi, G. Kautzmann, Y. Leclercq, S. Waniorek, and L. Williams, HIE ISOLDE alignment and monitoring system technical design and project status, Report No. CERN-ATS- 2012-269 (European Organization for Nuclear Research, Geneva, 2012).

[43] R. Laxdal, K. Fong, A. Mitra, T. Ries, I. Sekachev, G. Stanford, and V. Zviagintsev, Cryogenic, magnetic and RF performance of the ISAC-II medium beta cryomodule at TRIUMF, in Proceedings of the 21st Particle Accelerator Conference, Knoxville, U.S.A, 2005 (IEE, Piscataway, 2005), pp. 3191-3193.

[44] T. Xu et al., Progress of FRIB SRF production, in Proceedings of the 18th International Conference of RF Superconductivity, Lanzhou, China, 2017 (JACoW, Geneva, 2017), pp. 345-352.

[45] M. Champion, SNS RF system performance and operation, in Proceedings of 22nd Particle Accelerator Conference, Albuquerque, U.S.A, 2017 (IEEE, New York, 2017), pp. 3792-3794.

[46] R. Rybaniec et al., FPGA based RF and piezo controllers for SRF cavities in CW mode, in Proceedings of the 20th Real Time Conference (RT), Padova, Italy, 2016 (IEEENPS, 2016), pp. 1-2.

[47] V. Shiltsev, Observations of Random Walk of the Ground in Space in Time, Phys. Rev. Lett. 104, 238501 (2010).

[48] M. G. Minty and F. Zimmermann, Measurement and Control of Charged Particle Beams (Springer, 2003).

[49] P. Ostroumov et al., Beam commissioning in the first superconducting segment of the facility for rare isotope beams, Phys. Rev. ST. Accel. Beams 22, 080101 (2019).

[50] N. Chauvin, N. Bazin, S. Chel, L. Du, and J. Plouin, Beam dynamics errors studies for the IFMIF-DONES SRFLINAC, in Proceedings of the 10th International Particle Accelerator Conference, Melbourne, Australia, 2019 (JACoW, Geneva, 2019), pp. 3103-3105.

[51] S. Sato, Z. Igarashi, S. Lee, T. Tomisawa, F. Hiroki, J. Kishiro, M. Ikegami, Y. Kondo, K. Hasegawa, A. Ueno, T. Toyama, N. Kamikubota, K. Nigorikawa, M. Tanaka, and H. Yoshikawa, Sysgtematic calibration of beam position monitor in the high intensity proton accelerator (J-PARC) Linac, in Proceedings of 22nd International Linear Accelerator Conference, Lübeck, Germany, 2004 (JACoW, Geneva, 2004), pp. 429-431. 